\title{
Pairing mechanism in Fe-based superconductors
}

\author{
Andrey Chubukov \\ Department of Physics, University of Wisconsin, Madison, Wisconsin 53706, USA
}

(Dated: August 17, 2011)

\begin{abstract}
I review recent works on the symmetry and the structure of the superconducting gap in Fe-based superconductors and on the underlying pairing mechanism in these systems. The experimental data on superconductivity show very rich behavior, with potentially different symmetry of a superconducting state for different compositions of the same material. The variety of different pairing states raised the issue whether the physics of Fe-based superconductors is model-dependent or is universal, governed by a single underlying pairing mechanism. I argue that the physics is universal and that all pairing states obtained so far can be understood within the same universal pairing scenario and are well described by the effective low-energy model with a small number of input parameters.
\end{abstract}




\section{INTRODUCTION}

The discovery, in 2008, of superconductivity in Fe-based pnictides [1] (binary compounds of the elements from the 5th group: N, P, As, Sb, Bi) was, arguably, among the most significant breakthroughs in condensed matter physics during the past decade. A lot of efforts by the condensed-matter community have been devoted in the few years after the discovery to understand normal state properties of these materials, the pairing mechanism, and the symmetry and the structure of the pairing gap.

The family of $\mathrm{Fe}$-based superconductors (FeSCs) is already quite large and keeps growing. It includes various Fe-pnictides such as 1111 systems RFeAsO ( $R=$ rare earth element) [1-4], 122 systems $\mathrm{XFe}_{2} \mathrm{As}_{2}(\mathrm{X}=$ alkaline earth metals) [5-7], 111 systems like LiFeAs [9], and also

Fe-chalcogenides (Fe-based compounds with elements from the 16th group: S, Se, Te) such as $\mathrm{FeTe}_{1-x} \mathrm{Se}_{x} \quad$ [10] and $\mathrm{AFe}_{s} \mathrm{Se}_{2}(A=K, R b, C s)$ [11,12].

Parent compounds of FeSCs are metals, in distinction to cuprate superconductors for which parent compounds are Mott insulators. Still, in similarity with the cuprates, in most cases these parent compounds are antiferromagnetically ordered [13]. Because electrons which carry magnetic moments still travel relatively freely from site to site, the magnetic order is often termed as a spin-density-wave (SDW), by analogy with e.g., antiferromagnetic $C r$, rather than "Heisenberg antiferromagnetism" - the latter term is reserved for systems in which electrons are "nailed down" to particular lattice sites by very strong Coulomb repulsion.

Superconductivity (SC) in FeSCs emerges upon either hole or electron doping (see Fig. 1), but can also be induced by pressure or by isovalent replacement of one pnictide element by another, e.g., As by P (Ref. [8]). In some systems, like LiFeAs [9] and LaFePO [14], SC emerges already at zero doping, instead of of a magnetic order.

The magnetism, the electronic structure, the normal state properties of FeSCs, and the interplay between FeSCs and cuprate superconductors have been reviewed in several recent publications [15-25]. This review is an attempt to summarize our current understanding of the pairing mechanism and the symmetry and the structure of the pairing gap at various hole and electron dopings.

The phenomenon of SC has a long history. SC has been discovered by Kamerlingh Onnes exactly a century ago [26]). It has been explained in general terms nearly fifty years later, in 


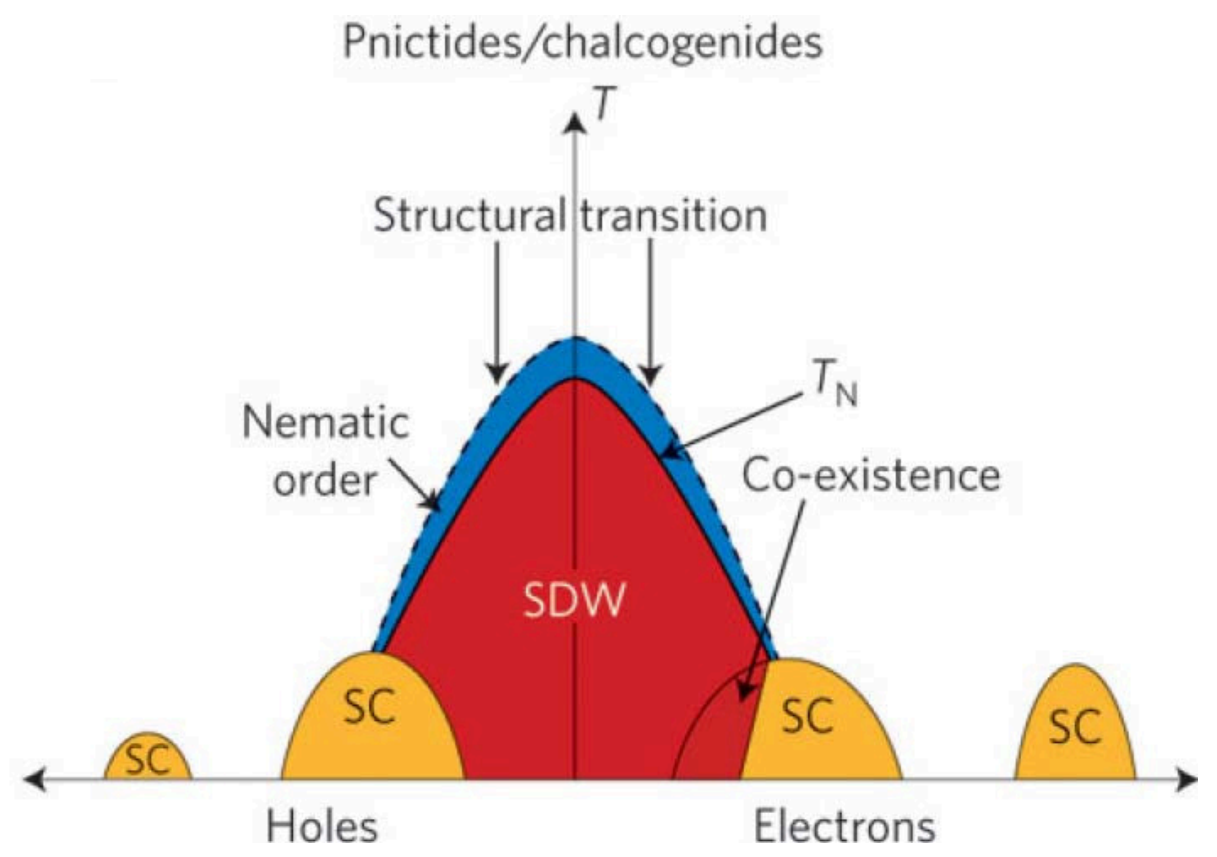

FIG. 1: Schematic phase diagram of Fe-based pnictides upon hole or electron doping. In the shaded region, superconductivity and antiferromagnetism co-exist. Not all details/phases are shown. Superconductivity can be initiated not only by doping but also by pressure and/or isovalent replacement of one pnictide element by another [8]. Nematic phase at $T>T_{N}$ is subject of debates. Superconductors at large doping are $\mathrm{KFe}_{2} \mathrm{As}_{2}$ for hole doping $[40,41]$ and $\mathrm{A}_{x} \mathrm{Fe}_{2-y} \mathrm{Se}_{2}(\mathrm{~A}=\mathrm{K}, \mathrm{Rb}$, Cs) for electron doping $[11,12]$. Whether superconductivity in pnictides exists at all intermediate dopings is not clear yet. From Ref. [24].

1957, by Bardeen, Cooper, and Schrieffer (BCS), who demonstrated that an arbitrary weak attractive interaction between low-energy fermions is sufficient to pair them into a bound state. At weak coupling, paired fermions immediately form Bose-Einstein condensate and behave as one single macroscopic quantum object and move coherently under the applied electric field, i.e superconduct. In $d$-dimensional electronic systems low-energy fermonic states are located, in momentum space, near particular $d-1$ dimensional surfaces, called 
Fermi surfaces (FS) on which fermionic energy is zero relative to the chemical potential. At weak/moderate coupling, the pairing problem is confined to a near vicinity of a FS. The interaction between fermions is generally non-singular with respect to variations of the distance to the FS and can be approximated by its value right on the FS.

What causes the attraction between fermions is a more subtle question, and the nature and the origin of the pairing glue have been the subject of great debates in condensed-matter community over the last 50 years. BCS attributed the attraction between fermions to the underlying interaction between electrons and phonons [27] (the two electrons effectively interact with each other by emitting and absorbing the same phonon which then serves as a glue which binds electrons into pairs). Electron-phonon mechanism has been successfully applied to explain $\mathrm{SC}$ in a large variety of materials, from $H g$ and $A l$ to recently discovered and extensively studied $M g B_{2}$ with the transition temperature $T_{c}=39 K \quad$ [28]. Nonphononic mechanisms of the pairing have also been discussed, most notably in connection with superfluidity in ${ }^{3} \mathrm{He}$ [29], but didn't become the mainstream before the discovery of SC in $\mathrm{LaBaCuO}$ in 1986 [30]. That discovery, and subsequent discoveries of superconductivity at higher $T_{c}$ in other cuprates signaled the beginning of the new era of "high-temperature superconductivity" to which FeSCs added a new avenue with quite high traffic over the last three years.

Superconductivity is quite robust phenomenon. It has been known from early 60th [31] that in isotropic systems the equation for superconducting $T_{c}$ factorizes if one expands the interaction between the two fermions in partial components corresponding to interactions in the subspaces with a given angular momentum of the two interacting fermions $l=0,1,2,3$, etc [in spatially isotropic systems $l=0$ component is called $s$-wave, $l=1$ component is called $p$-wave, $l=2$ component is called $d$-wave, and so on]. If just one component with some $l$ is attractive, the system undergoes a $\mathrm{SC}$ transition at some temperature $T=T_{c}$. For phonon-mediate superconductors, $s$-wave superconductivity is the most likely outcome. In the cuprates, however, the pairing symmetry has been firmly established as $d$-wave. The vast majority of researches believe that such pairing is not caused by phonons and emerges instead due to screened Coulomb interaction between electrons. The screened Coulomb interaction $U(r)$ is constant and repulsive at short distances but has a complex dependence on $r$ at large distances and may develop an attractive component at some $l$. One solid reason for the attraction, at least at large $l$, has been identified by Kohn and Luttinger back in 
1965 (Ref. [32]).

In lattice systems, angular momentum is no longer a good quantum number, and the equation for $T_{c}$ only factorizes between different irreducible representations of the lattice space group. In tetragonal systems, which include both cuprates and FeSCs, there are four one-dimensional irreducible representations $A_{1 g}, B_{1 g}, B_{2 g}$, and $A_{2 g}$ and one two-dimensional representation $E_{2 g}$. Each representation has infinite set of eigenfunctions. The eigenfunctions from $A_{1 g}$ are invariant under symmetry transformations in a tetragonal lattice: $x \rightarrow-x, y \rightarrow-y, x \rightarrow y$, the eigenfunctions from $B_{1 g}$ change sign under $x \rightarrow y$, and so on. If a superconducting gap has $A_{1 g}$ symmetry, it is often called $s$-wave because the first eigenfunction from $A_{1 g}$ group is just a constant in momentum space (a $\delta$-function in real space). If the gap has $B_{1 g}$ or $B_{2 g}$ symmetry, it is called $d$-wave $\left(d_{x^{2}-y^{2}}\right.$ or $d_{x y}$, respectably), because in momentum space the leading eigenfunctions in $B_{1 g}$ and $B_{2 g}$ are $\cos k_{x}-\cos k_{y}$ and $\sin k_{x} \sin k_{y}$, respectively, and these two reduce to $l=2$ eigenfunctions $\cos 2 \theta$ and $\sin 2 \theta$ in the isotropic limit.

In the cuprates, the superconducting gap has been proved experimentally to have $B_{1 g}$ symmetry [33]. This gap symmetry appears quite naturally in the cuprates, in the doping range where they are metals, if one assumes that the glue that binds fermions together is a spin-fluctuation exchange rather than a phonon (see Fig.2). The notion of a spin fluctuation is actually nothing but the convenient way to describe multiple Coulomb interactions between fermions. It is believed, although not proved rigorously, that in systems located reasonably close to a magnetic instability, the fully screened Coulomb interaction between fermions can be approximated by an effective interaction in which fermions exchange quanta of their collective fluctuations in the spin channel. That $B_{1 g}$ gap is selected is not a surprise because such gap $\Delta(k) \propto \cos _{x}-\cos k_{y}$ changes sign not only under $k_{x} \rightarrow k_{y}$ but also between $\mathbf{k}$ and $\mathbf{k}^{\prime}=\mathbf{k}+\mathbf{Q}$ where $\mathbf{Q}=(\pi, \pi)$ is the momenta at which spin fluctuationmediated pairing interaction $U\left(\mathbf{k}, \mathbf{k}^{\prime}\right)$ is peaked. This sign change is the crucial element for any electronic mechanism of superconductivity because one needs to extract an attractive (negative) component from repulsive (positive) screened Coulomb interaction. For $B_{1 g}$ gap such a component is $\int d \mathbf{k} d \mathbf{k}^{\prime} \Delta(k) U\left(\mathbf{k}, \mathbf{k}^{\prime}\right) \Delta\left(k^{\prime}\right)$, and the integral obviously has a negative value when $U\left(\mathbf{k}, \mathbf{k}^{\prime}\right)$ is peaked at $(\pi, \pi)$.

In FeSCs, magnetism and superconductivity are also close neighbors on the phase diagram, and it has been proposed $[34,35]$ at the very beginning of the $F e$ era that the pairing 


\section{Cuprates}

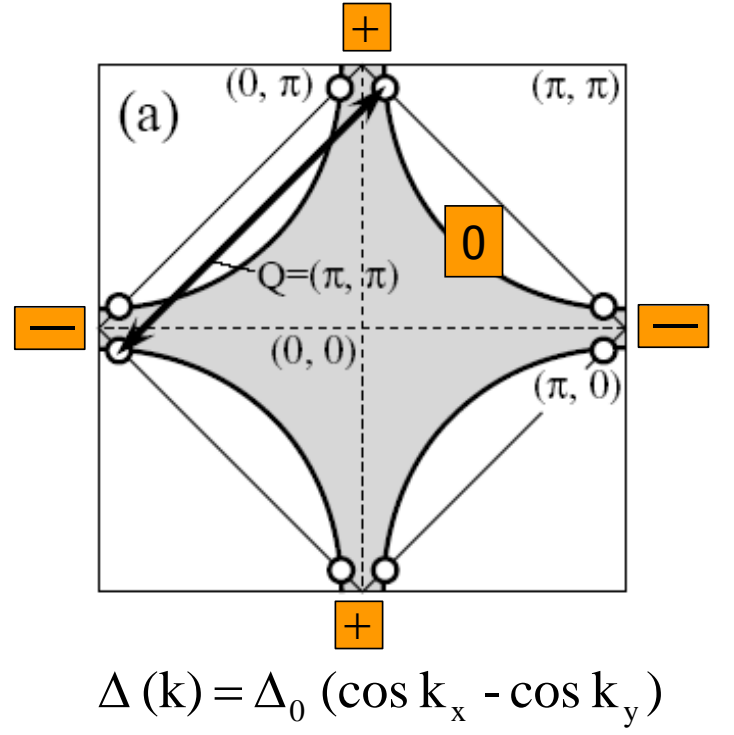

Pnictides

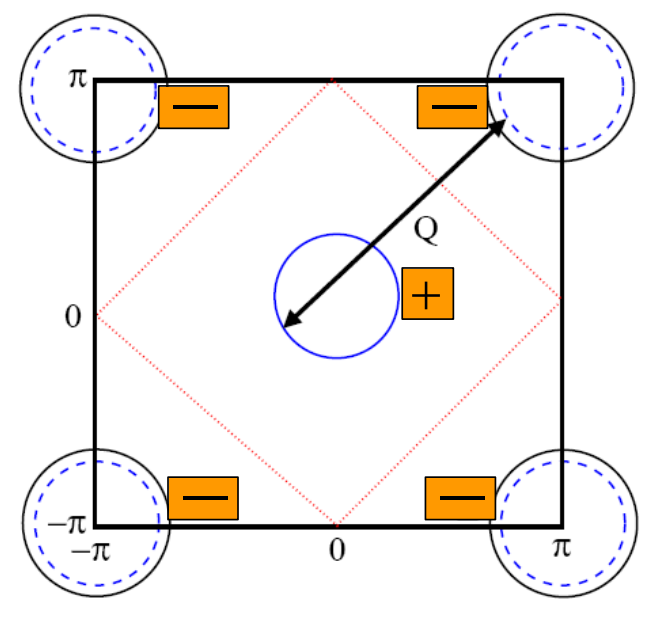

$\Delta(k=0)=\Delta_{0}, \Delta(k=\pi, \pi)=-\Delta_{0}$

FIG. 2: A comparison of the pairing state from spin-fluctuation exchange in cuprate SCs and in FeSCs. In the cuprates (left panel) the FS is large, and antiferromagnetic $\mathbf{Q}=(\pi, \pi)$ connects points on the same FS. Because spin-mediated interaction is positive (repulsive), the gap must change sign between FS points separated by $\mathbf{Q}$. As the consequences, the gap changes sign twice along the FS. This implies a $d$-wave gap symmetry. In FeSCs (left panel) scattering by $\mathbf{Q}$ moves fermions from one FS to the other. In this situation, the gap must change sign between different FS, but to first approximation remains a constant on a given FS. By symmetry, such a gap is an $s$-wave gap. It is called $s^{+-}$because it changes sign between different FSs

mechanism in FeSCs is also a spin-fluctuation exchange. However, the geometry of lowenergy states in FeSCs and in the cuprates is different, and in most FeSCs the momentum Q connects low-energy fermionic states near the center and the corner of the Briilouin zone (see Fig.2). A simple experimentation with trigonometry then tell us that the SC gap $\Delta(k)$ must be symmetric with respect to $k_{x} \rightarrow k_{y}$ and $k_{x} \rightarrow-k_{x}$, but still must change sign under 
$\mathbf{k} \rightarrow \mathbf{k}+\mathbf{Q}$. Such gap belongs to $A_{1 g}$ representation, but it only has contributions from a particular subset of $A_{1 g}$ states with the form $\cos k_{x}+\cos k_{y}, \cos 3 k_{x}+\cos \left(3 k_{y}\right)$, etc which all change sign under $\mathbf{k} \rightarrow \mathbf{k}+\mathbf{Q}$. Such gap is generally called an extended $s$-wave gap, or $s^{+-}$gap.

Majority of researches do believe that in weakly/moderately doped FeSCs the gap does have $s^{+-}$symmetry. However, numerous studies of superconductivity in FeSCs over the last three years demonstrated that the physics of the pairing is more involved than it was originally thought because of multi-orbital/multi-band nature of low-energy fermionic excitations in FeSCs (see below). It turns out that both the symmetry and the structure of the pairing gap result from rather non-trivial interplay between spin-fluctuation exchange, intraband Coulomb repulsion, and momentum structure of the interactions. In particular, an $s^{ \pm}$wave gap can be with or without nodes, depending on the orbital content of low-energy excitations. In addition, the structure of low-energy spin fluctuations evolves with doping, and the same spin-fluctuation mechanism that gives rise to $s^{+-}$gap at small/moderate doping in a particular material can give rise to a $d$-wave gap at strong hole or electron doping.

There is more uncertainly on the theory side. In addition to spin fluctuations, FeSCs also possess charge fluctuations whose strength is the subject of debates. There are proposals [36, 37] that in multi-orbital FeSCs charge fluctuations are strongly enhanced because the system is reasonably close to a transition into a state with an orbital order (e.g., a spontaneous symmetry breaking between the occupation of different orbitals). A counter-argument is that orbital order does not develop on its own but is induced by a magnetic order [38]. If charge fluctuations are relevant, one should consider, in addition to spin-mediated pairing interaction, also the pairing interaction mediated by charge fluctuations. The last interaction can give rise to a conventional, sign-preserving $s$-wave pairing [36]. A "p-wave" gap scenario (a gap belonging to $E_{2 g}$ representation) has also been put forward [39].

From experimental side, $s$-wave gap symmetry is consistent with ARPES data on moderately doped $\mathrm{KFe}_{2} \mathrm{As}_{2}$ and $\mathrm{BaFe}_{2}\left(\mathrm{As}_{1-x} \mathrm{P}_{x}\right)_{2}$, which detected only a small variation of the gap along the FSs centered at $(0,0)$ (Ref. [42]), and with the evolution of the tunneling data in a magnetic field [98. However, for heavily hole-doped $\mathrm{KFe}_{2} \mathrm{As}_{2}$ various experimental probes [41] indicate the presence of gap nodes, which for the FS geometry in these materials [40] are consistent with a $d$-wave gap. For the doping range where the gap is very likely an $s$-wave, the data on some FeSCs were interpreted as evidence for the full gap [44-47, 
while the data for other FeSCs were interpreted as evidence that the gap has nodes $[48,49]$ or deep minima [50-52]. In addition, recent nuclear magnetic resonance (NMR) experiments on LiFeAs have been interpreted in favor of a $p$-wave gap [43].

In this paper, I argue that all these seemingly very different gap structures (with the exception of a $p$-wave), actually follow quite naturally from the same underlying physics idea that FeSCs can be treated as moderately interacting itinerant fermionic systems with multiple FS sheets and effective four-fermion intra-band and inter-band interactions in the band basis. I introduce the effective low-energy model with small numbers of input parameters [66] and use it to study the doping evolution of the pairing in hole and electron-doped FeSCs. It has been argued [66] that various approaches based on underlying microscopic model in the orbital basis reduce to this model at low energies.

The paper is organized as follows. In Sec. II I discuss general aspects of the pairing in FeSCs. I briefly review the band structure of FeSCs and show that it contains several bands of low-energy excitations. I then present generic symmetry considerations of the pairing in a multi-band superconductor. I show that a "conventional wisdom" that an s-wave gap is nodeless along the FSs, d-wave gap has 4 nodes, etc, has only limited applicability in multiband superconductors, and there are cases when the gap with four nodes has an $s$-wave symmetry, and the gap without nodes has a $d$-wave symmetry. In Sec. III I discuss the interplay between intra-band and inter-band interactions, first for a toy two-pocket model and then for realistic multi-pocket models, and set the conditions for an attraction in an $s$-wave or a $d$-wave channel. I consider 5-orbital model with local interactions, convert it into a band basis, and argue that for most of input parameters the bare interaction is repulsive in all channels due to strong intra-pocket Coulomb repulsion. In Sec. IV I discuss the ways to overcome Coulomb repulsion. I review random phase approximation (RPA) and renormalization group $(\mathrm{RG})$ approaches and show that magnetic fluctuations enhance interpocket interaction, if this interaction is positive, what gives rise to an attraction in both $s^{ \pm}$and $d_{x^{2}-y^{2}}$ channels. I briefly discuss $s^{++}$pairing, which emerges if input parameters are such that inter-pocket interaction is negative. In Sec.V I use the combination of RPA and leading angular harmonic approximation (LAHA) to analyze the pairing in $s$ - and $d$-wave channels at different dopings. I show that magnetically-mediated pairing leads to (i) an $s^{ \pm}$superconductivity with nodes on electron pockets for moderate electron doping, (ii) an $s^{ \pm}$superconductivity without nodes for moderate hole dopings, (iii) a nodeless $d$-wave 

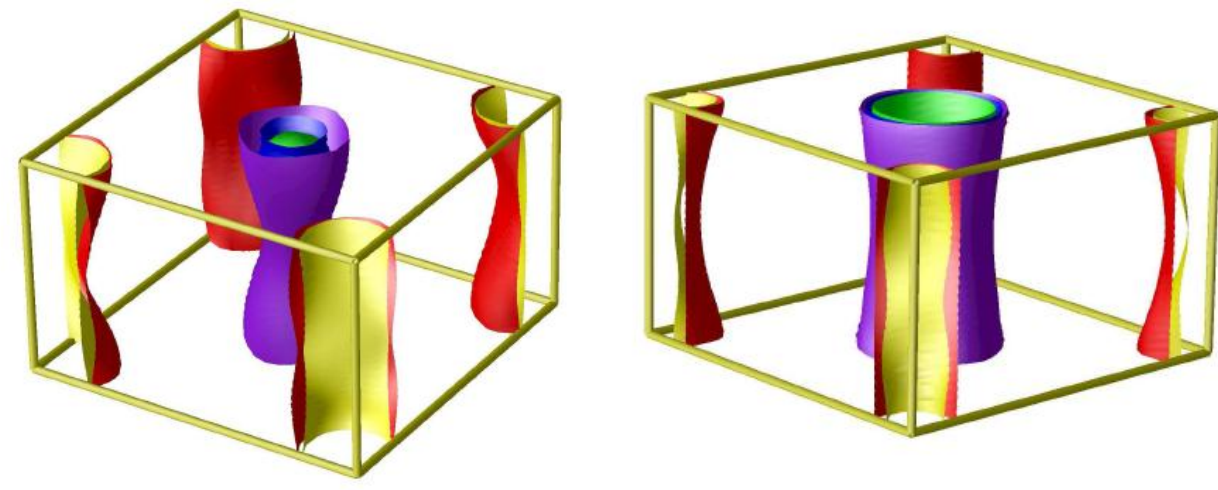

FIG. 3: The electronic structure of FeSCs. In weakly and moderately electron-doped materials (left panel) the FS consists of quasi-2D warped cylinders centered at $(0,0)$ and $(\pi, \pi)$ in a $2 \mathrm{D}$ cross-section. The ones near $(0,0)$ are hole pockets (filled states are outside cylinders), the ones near $(\pi, \pi)$ are electron pockets (filled states are inside cylinders) There also exists a quasi-3D hole pocket near $k_{z}=\pi$. In hole-doped FeSCs the electronic structure is very similar, but 3D hole pocket becomes quasi-2D warped hole cylinder. From Ref. [23].

superconductivity for strong electron doping (except, possibly, small nodal regions near $k_{z}=\pi / 2$ ), and (iv) a nodal $d$-wave superconductivity for strong hole doping. I briefly review the experimental situation in Sec. VII and present concluding remarks in Sec. VII. I list separately the summary points and the list of future issues.

\section{GENERIC ASPECTS OF PAIRING IN FESCS}

\section{A. The electronic structure}

The electronic structure of FeSCs at low energies is rather well established by ARPES [53] and quantum oscillation measurements [54]. In weakly and moderately electron-doped ma- 
terials, like $\mathrm{BaFe}_{1-x} \mathrm{Co}_{x} \mathrm{Fe}_{2} \mathrm{As}_{2}$ the FS contains several quasi-2D warped cylinders centered at $k=(0,0)$ and $k=(\pi, \pi)$ in a $2 \mathrm{D}$ cross-section, and may also contain a quasi-3D pocket near $k_{z}=\pi$ (Fig.3). The fermionic dispersion is electron-like near the FSs at $(\pi, p i)$ (filled states are inside a FS) and hole-like near the FSs centered at $(0,0)$ (filled states are outside a FS). In heavily electron-doped FeSCs, like $\mathrm{A}_{x} \mathrm{Fe}_{1-y} \mathrm{Se}_{2}(\mathrm{~A}=\mathrm{K}, \mathrm{Rb}, \mathrm{Cs})$, only electron pockets remain, according to recent ARPES studies. [11] In weakly and moderately holedoped FeSCs, like $\mathrm{Ba}_{1-x} \mathrm{~K}_{x} \mathrm{Fe}_{2} \mathrm{As}_{2}$, the electronic structure is similar to that at moderate electron doping, however the spherical FS becomes the third quasi 2D hole FS centered at $(2 \pi, 0)=(0,0)$. In addition, new low-energy hole states likely appear around $(\pi, \pi)$ and squeeze electron pockets [55]. At strong hole doping, electron FSs disappear and only only hole FSs are present [40] These electronic structures agree well with first-principle calculations $[17,56,57]$, which is another argument to treat FeSCs as itinerant fermionic systems. The measured FS reflects the actual crystal structure of FeSCs in which there are two non-equivalent positions of a pnictide above and below an $F e$ plane, and, as a result, there are two $F e$ atoms in the unit cell (this actual Brillouin zone (BZ) is called "folded BZ"). From theory perspective, it would be easier to work in the BZ which contains only one $\mathrm{Fe}$ atom in the unit cell (this theoretical BZ is called "unfolded BZ"). I illustrate the difference between folded and unfolded BZ in Fig.4. In general, only folded BZ is physically meaningful. However, if by some reason a potential from a pnictogen (or chalcogen) can be neglected, the difference between the folded and the unfolded BZ becomes purely geometrical: the momenta $\tilde{k}_{x}$ and $\tilde{k}_{y}$ in the folded $\mathrm{BZ}$ are linear combinations of $k_{x}$ and $k_{y}$ in the unfolded BZ: $\tilde{k}_{x}=k_{x}+k_{y}, \tilde{k}_{y}=k_{x}-k_{y}$. In this situation, folded and unfolded BZ become essentially equivalent.

Most of the existing theory works on the pairing mechanism and the structure of the $\mathrm{SC}$ gap analyze the pairing problem in the unfolded BZ, where which two hole pockets are centered at $(0,0)$ and one at $(\pi, p i)$, and the two electron pockets are are at $(0, \pi)$ and $(\pi, 0)$. It became increasingly clear recently that the interaction via a pnictogen/chalcogen and also 3D effects do play some role for the pairing, particularly in strongly electron-doped systems. [58,59] However, it is still very likely that the key aspects of the pairing in FeSCs can be understood by analyzing a pure 2D electronic structure with only Fe states involved. Below I assume that this is the case and consider a 2D model in the unfolded BZ with hole FSs near $(0,0)$ and $(\pi, p i)$ and electron FSs at $(0, \pi)$ and $(\pi, 0)$. 

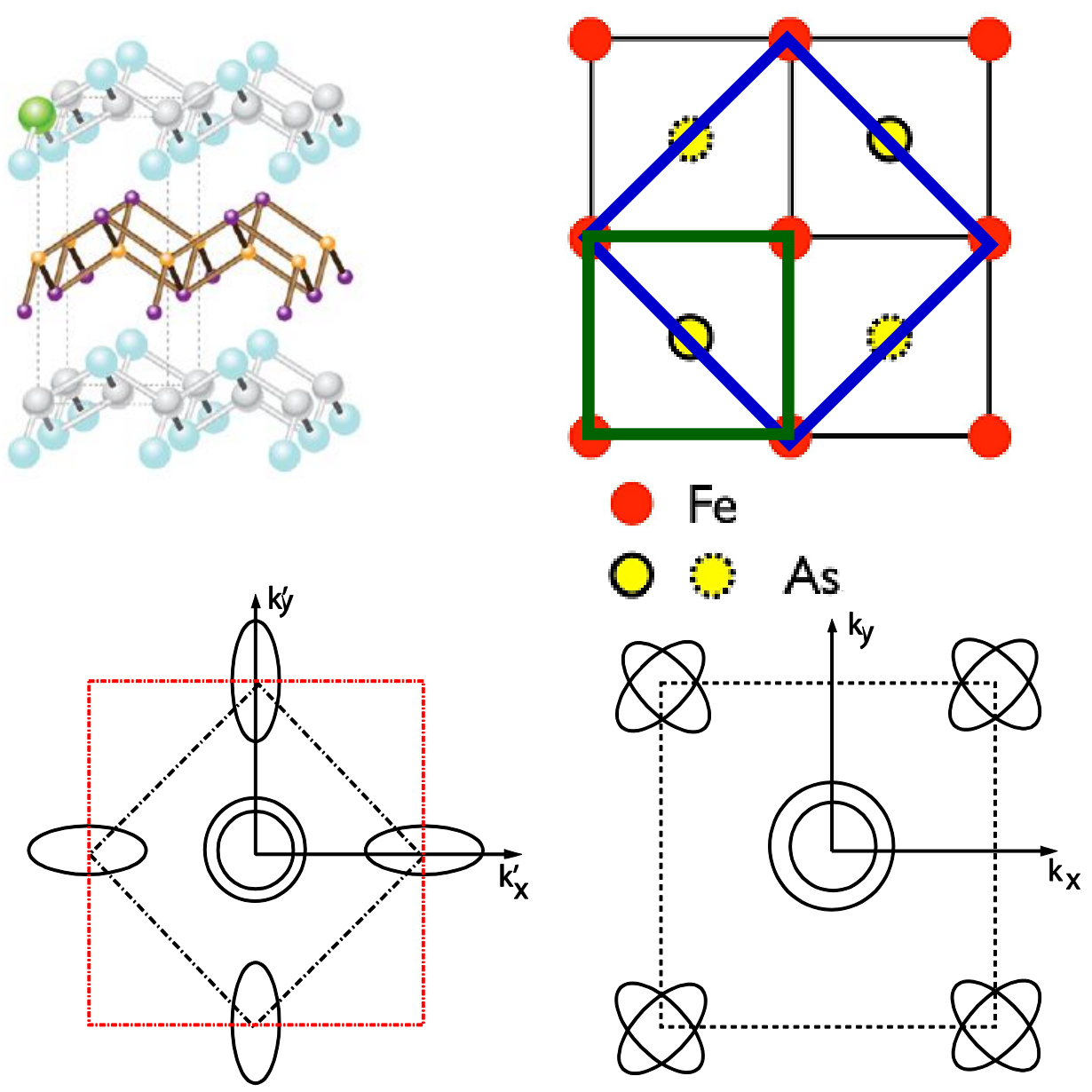

FIG. 4: Upper panel: 3D electronic structure of LaOFeAs (left) and its 2D cross-section (left). In only Fe states are considered, an elementary cell contains one Fe atom (green). The actual unit cell (blue) contains two Fe atoms because of two non-equivalent positions of a pnictide above and below the Fe plane. Lower panel - the location of hole and electron FSs in a 2D cross section in the folded BZ (two Fe/cell, right) and in the unfolded BZ (one Fe/cell, left). From Refs. [60], $[61](\mathrm{b})$ and $[62] \mathrm{b}$.

\section{B. The structure of $s$-wave and $d$-wave gaps in a multi-band SC}

I now use the multi-band electronic structure as input and consider the pairing problem at weak coupling. I show that an $s$-wave gap generally has angle dependence on electron FSs and may even has nodes, while a d-wave gap which is normally assumed to have nodes, may in fact be nodeless on the same electron FSs. 


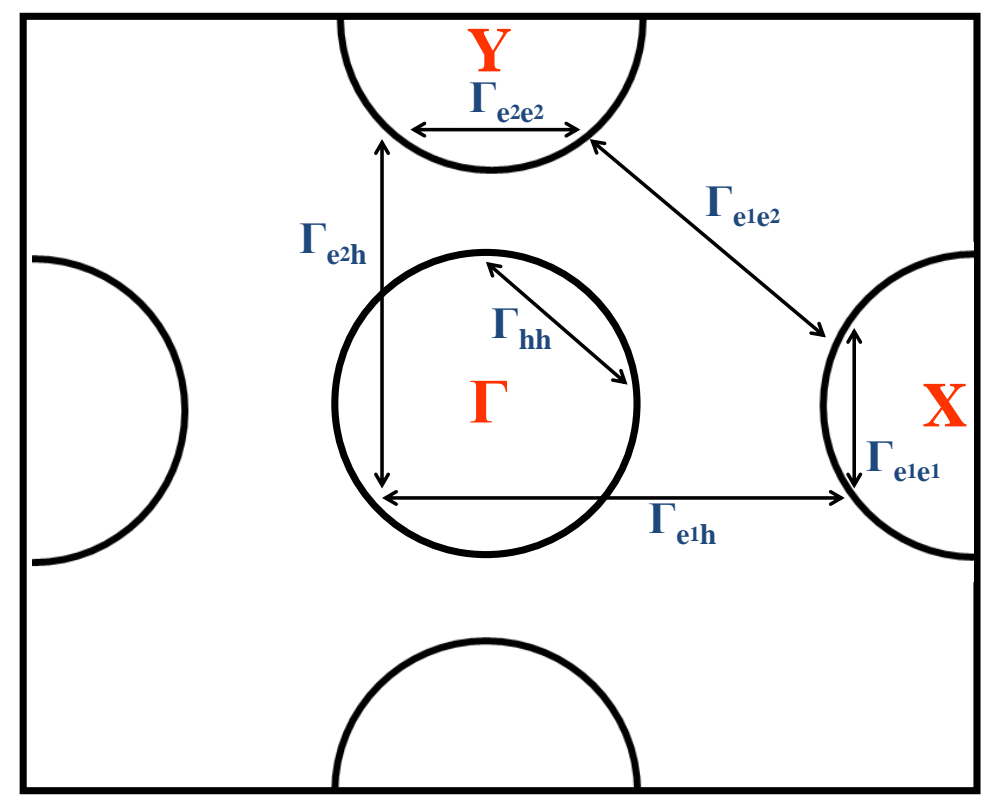

FIG. 5: Intra-pocket and inter-pocket interactions in 4-band 2D model for FeSCs. For simplicity, only one hole FS is shown. $\Gamma, X$, and $Y$ points are $(0,0),(\pi, 0)$, and $(0, \pi)$, respectively. $\Gamma_{h h}$ is the interaction within hole pocket, $\Gamma_{e_{1} h}$ and $\Gamma_{e_{2} h}$ are the interactions between hole and one of electron pockets, and $\Gamma_{e_{1} e_{1}}, \Gamma_{e_{2}, e_{2}}$ (not shown) and $G a m m a_{e_{1} e_{2}}$ are intra-pocket and inter-pocket interactions involving the two electron pockets. Each interaction contains $s$-wave and $d$-wave component, and even for $s$-wave $\Gamma_{e_{i} h}$ and $\Gamma_{e_{i} e_{j}}$ depend on the angles along electron FSs. From Ref. [64].

A generic low-energy BCS-type model in the band basis is described by

$$
\mathcal{H}=\sum_{i, \mathbf{k}} \epsilon_{i}(\mathbf{k}) a_{i \mathbf{k}}^{\dagger} a_{i \mathbf{k}}+\sum_{i, j, \mathbf{k}, \mathbf{p}} \Gamma_{i, j}(\mathbf{k}, \mathbf{p}) a_{i \mathbf{k}}^{\dagger} a_{i-\mathbf{k}}^{\dagger} a_{j \mathbf{p}} a_{j-\mathbf{p}}
$$

The quadratic term describes low-energy excitations near hole and electron FSs, labeled by $i$ and $j$, and the four-fermion term describes the scattering of a pair $(k \uparrow,-k \downarrow)$ on the FS $i$ to a pair $(p \uparrow,-p \downarrow)$ on the FS $j$. These interactions are either intra-pocket interactions (hole-hole $\Gamma_{h_{i} h_{i}}$ or electron-electron $\Gamma_{e_{i} e_{i}}$ ), or inter-pocket interactions (hole-electron $\Gamma_{e_{j} h_{i}}$, hole-hole $\Gamma_{h_{i} \neq h_{j}}$, and electron-electron $\Gamma_{e_{i} \neq e_{j}}$ ). I illustrate this in Fig.5.

Assume for simplicity that the frequency dependence of $\Gamma$ can be neglected and lowenergy fermions are Fermi-liquid quasiparticles with Fermi velocity $v_{k_{F}}$. In this situation, the gap $\Delta(k)$ also doesn't depend on frequency, and the linearized gap equation becomes 
the eigenfunction/eigenvalue problem:

$$
\lambda_{i} \Delta_{i}(k)=-\int \frac{d p_{\|}}{4 \pi^{2} v_{\mathbf{p}_{F}}} \Gamma\left(\mathbf{k}_{F}, \mathbf{p}_{F}\right) \Delta_{i}(p)
$$

where $\Delta_{i}$ are eigenfunctions and $\lambda_{i}$ are eigenvalues. The system is unstable towards pairing if one or more $\lambda_{i}$ are positive. The corresponding $T_{c, i}$ scale as $T_{c, i}=\Lambda_{i} e^{-1 / \lambda_{i}}$. Although $\Lambda_{i}$ are generally different for different $i$, the exponential dependence on $1 / \lambda_{i}$ implies that, most likely, the solution with the largest positive $\lambda_{i}$ emerges first and establish the pairing state, at least immediately below $T_{c}$.

Like we discussed in the Introduction, the vertex $\Gamma(k, p)$ can be decomposed into representations of the tetragonal space group (one-dimensional representations are $A_{1 g}, B_{1 g}$, $B_{2 g}$, and $A_{2 g}$ ). Basis functions from different representations do not mix, but each contains infinite number of components. For example, $s$-wave pairing corresponds to fully symmetric $A_{1 g}$ representation, and the $s$-wave $\left(A_{1 g}\right)$ component of $\Gamma(k, p)$ can be quite generally expressed as

$$
\Gamma^{(1 g)}(k, p)=\Gamma_{s}(k, p)=\sum_{m, n} A_{m n}^{s} \Psi_{m}^{s}(k) \Psi_{n}^{s}(p)
$$

where $\Psi_{m}^{s}(k)$ are the basis functions of the $\mathrm{A}_{1 g}$ symmetry group: $1, \cos k_{x} \cos k_{y}, \cos k_{x}+$ $\cos _{y}$, etc, and $A_{m n}^{s}$ are coefficients. Suppose that $k$ belongs to a hole FS and is close to $k=0$. Expanding any wave function with $\mathrm{A}_{1 g}$ symmetry near $k=0$, one obtains along $|\mathbf{k}|=k_{F}$,

$$
\Psi_{m}^{s}(k)=a_{m}+b_{m} \cos 4 \phi_{k}+c_{m} \cos 8 \phi_{k}+\ldots
$$

where $\phi_{k}$ is the angle along the hole FS (which is not necessary a circle). Similarly, for $B_{1 g}$ representation the wavefunctions are $\cos k_{x}-\cos k_{y}, \cos 2 k_{x}-\cos 2 k_{y}$, etc, and expanding them near $k=0$ one obtains

$$
\Psi_{m}^{d}(k)=a_{m}^{*} \cos 2 \phi_{k}+b_{m}^{*} \cos 6 \phi_{k}+c_{m}^{*} \cos 10 \phi_{k}+\ldots
$$

There are no fundamental reasons to expect that $b_{m}, c_{m}$ or $b_{m}^{*}, c_{m}^{*}$ are much smaller than $a_{m}$ or $a_{m}^{*}$, but sub-leading terms are often small numerically. The known example is the numerical smallness of $\cos (2+4 n) \phi$ components with $n \geq 1$ of the $d_{x^{2}-y^{2}}$-wave gap in the spin-fluctuation scenario for the cuprates [63] (meaning that a d-wave gap can be reasonably well approximated by $\cos 2 \phi$ ). Taking this example as circumstantial evidence, I neglect all subleading terms, i.e., assume that $s$-wave interaction between fermions on the hole FSs can 
be approximated by an angle-independent $\Gamma_{h_{i} h_{j}}^{s}(k, p)=U_{h_{i} h_{j}}\left(h_{i}\right.$ label different hole FSs), while $d$-wave $\left(B_{1 g}\right)$ interaction can be approximated by $\Gamma_{h_{i} h_{j}}^{d}(k, p)=\tilde{U}_{h_{i} h_{j}} \cos 2 \phi_{k} \cos 2 \phi_{p}$.

The situation changes, however, when we consider the pairing component involving fermions from electron FSs. Suppose that $k$ are still near the center of the BZ, but $p$ are near one of the two electron FSs, say the one centered at $(0, \pi)$. Consider all possible $\Psi_{n}(p)$ with $A_{1 g}$ symmetry A simple experimentation with trigonometry shows that there are two different subsets of basis functions:

$$
\begin{aligned}
& \text { subset } I: 1, \cos p_{x} \cos p_{y}, \quad \cos 2 p_{x}+\cos 2 p_{y} \ldots \\
& \text { subset } I I: \cos p_{x}+\cos p_{y}, \quad \cos 3 p_{x}+\cos 3 p_{y} \ldots
\end{aligned}
$$

For a circular FS centered at $(0, \pi)$, the functions from subset I can be again expanded in series of $\cos 4 l \phi_{p}$ with integer $l$. The functions from subset II are different - they all vanish at $(0, \pi)$ and are expanded in series of $\cos \left(2 \phi_{p}+4 l \phi_{p}\right)$ (the first term is $\cos 2 \phi_{p}$, the second is $\cos 6 \phi_{p}$, etc). For elliptic $\mathrm{FS} \cos 4 l \phi_{p}$ and $\cos \left(2 \phi_{p}+4 l \phi_{p}\right)$ terms appear in both subsets. In both cases, the total

$$
\begin{aligned}
\Psi_{m}^{s}(p)= & \bar{a}_{m}+\bar{b}_{m} \cos 4 \phi_{p}+\bar{c}_{m} \cos 8 \phi_{p}+\ldots \\
& +\overline{\bar{a}}_{m} \cos 2 \phi_{p}+\overline{\bar{b}}_{m} \cos 6 \phi_{p}+\overline{\bar{c}}_{m} \cos 10 \phi_{k}+\ldots
\end{aligned}
$$

For the other electron $\mathrm{FS}, \Psi_{m}^{s}(p)$ is the same, but momentum components $p_{x}$ and $p_{y}$ are interchanged, hence the sign of all $\cos \left(2 \phi+4 l \phi_{p}\right)$ components changes.

Let's make the same approximation as before and neglect all components with $l>0$. Then

$$
\Psi_{m}^{s}(p)=\bar{a}_{m} \pm \overline{\bar{a}}_{m} \cos 2 \phi_{p}
$$

where the upper sign is for one electron FS and the lower for the other. It is essential that the angle-independent term and the $\cos 2 \phi_{p}$ term have to be treated on equal footing because each is the leading term in the corresponding series. Combing (8) with the fact that $\Psi_{m}^{s}(k)$ can be approximated by a constant, we obtain a generic form of the $s$-wave component of the interaction between fermions near hole and electron FSs

$$
\begin{aligned}
& \Gamma_{e_{1}, h_{i}}^{s}(k, p)=U_{e, h_{i}}\left(1+2 \alpha_{e, h} \cos 2 \phi_{p_{e 1}}+\ldots\right) \\
& \Gamma_{e_{2}, h_{i}}^{s}(k, p)=U_{e, h_{i}}\left(1-2 \alpha_{e, h} \cos 2 \phi_{p_{e 2}}+\ldots\right)
\end{aligned}
$$

where dots stand for $\cos 4 \phi_{k}, \cos 4 \phi_{p}, \cos 6 \phi_{p}$, etc terms. 
By the same reasoning, $s$-wave components of inter-pocket and intra-pocket interactions between fermions from electron FSs are

$$
\begin{aligned}
\Gamma_{e_{1}, e_{1}}^{s}(k, p)= & U_{e, e}\left(1+2 \alpha_{e e}\left(\cos 2 \phi_{k_{e 1}}+\cos 2 \phi_{p_{e 1}}\right)\right. \\
& +4 \beta_{e e} \cos 2 \phi_{k_{e 1}} \cos 2 \phi_{p_{e 1}}+\ldots \\
\Gamma_{e_{2}, e_{2}}^{s}(k, p)= & U_{e, e}\left(1-2 \alpha_{e e}\left(\cos 2 \phi_{k_{e 2}}+\cos 2 \phi_{p_{e 2}}\right)\right. \\
& +4 \beta_{e e} \cos 2 \phi_{k_{e 2}} \cos 2 \phi_{p_{e 2}}+\ldots \\
\Gamma_{e_{1}, e_{2}}^{s}(k, p)= & U_{e, e}\left(1+2 \alpha_{e e}\left(\cos 2 \phi_{k_{e 1}}-\cos 2 \phi_{p_{e 2}}\right)\right. \\
& -4 \beta_{e e} \cos 2 \phi_{k_{e 1}} \cos 2 \phi_{p_{e 2}}+\ldots
\end{aligned}
$$

Once the pairing interaction has the form of Eqs. (9) and (10), the gaps along the hole FSs are angle-independent (modulo $\cos 4 \phi$ terms), but the gaps along the two electron FSs are of the form

$$
\Delta_{e}^{(s)}(k)=\Delta_{e} \pm \bar{\Delta}_{e} \cos 2 \phi_{k}
$$

When $\bar{\Delta}_{e}$ is small compared to $\Delta_{e}$, the angle dependence is weak, but when $\left|\bar{\Delta}_{e}\right|>\left|\Delta_{e}\right|$, $s$-wave gaps have nodes at "accidental" values of $\phi$, which differ between the two electron FSs.

A similar consideration holds for $d_{x^{2}-y^{2}}$ gap. Within the same approximation of leading angular momentum harmonics, we have

$$
\begin{aligned}
& \Gamma_{e_{1}, h_{i}}^{d}(k, p)=\tilde{U}_{e, h_{i}} \cos 2 \phi_{h_{i}}\left(1+\tilde{\alpha}_{e, h} \cos 2 \phi_{p_{e 1}}\right)+\ldots \\
& \Gamma_{e_{2}, h_{i}}^{d}(k, p)=\tilde{U}_{e, h_{i}} \cos 2 \phi_{h_{i}}\left(-1+\tilde{\alpha}_{e, h} \cos 2 \phi_{p_{e 2}}\right)+\ldots
\end{aligned}
$$

and

$$
\begin{aligned}
\Gamma_{e_{1}, e_{1}}^{d}(k, p)= & \tilde{U}_{e, e}\left(1+2 \alpha_{e e}\left(\cos 2 \phi_{k_{e 1}}+\cos 2 \phi_{p_{e 1}}\right)\right. \\
& +4 \beta_{e e} \cos 2 \phi_{k_{e 1}} \cos 2 \phi_{p_{e 1}}+\ldots \\
\Gamma_{e_{2}, e_{2}}^{d}(k, p)= & \tilde{U}_{e, e}\left(1-2 \alpha_{e e}\left(\cos 2 \phi_{k_{e 2}}+\cos 2 \phi_{p_{e 2}}\right)\right. \\
& +4 \beta_{e e} \cos 2 \phi_{k_{e 2}} \cos 2 \phi_{p_{e 2}}+\ldots \\
\Gamma_{e_{1}, e_{2}}^{d}(k, p)= & \tilde{U}_{e, e}\left(-1-2 \alpha_{e e}\left(\cos 2 \phi_{k_{e 1}}-\cos 2 \phi_{p_{e 2}}\right)\right. \\
& +4 \beta_{e e} \cos 2 \phi_{k_{e 1}} \cos 2 \phi_{p_{e 2}}+\ldots
\end{aligned}
$$


The solution of the gap equation then yields the gap in the form

$$
\begin{aligned}
& \Delta_{h}^{(d)}(k)=\tilde{\Delta}_{h} \cos 2 \phi_{k} \\
& \Delta_{e}^{(d)}(k)= \pm \tilde{\Delta}_{e}+\overline{\tilde{\Delta}}_{e} \cos 2 \phi_{k} .
\end{aligned}
$$

Along the hole FS, the gap behaves as a conventional $d$-wave gap with 4 nodes along the diagonals. Along electron FSs, the two gaps differ in the sign of the angle-independent terms, and have in-phase $\cos 2 \phi$ oscillating components. When $\overline{\tilde{\Delta}}_{e}<<\tilde{\Delta}_{e}$ the two electron gaps are simply "plus" and "minus" gaps, but when $\overline{\tilde{\Delta}}_{e}>\tilde{\Delta}_{e}$, each has accidental nodes, again along different directions on the two electron FSs.

We see therefore that the geometry of the FSs in FeSCs affects the gap structure in quite fundamental way: because electron FSs are centered at the $k$ points which are not along BZ diagonals, $s$-wave gaps on these FSs have $\cos 2 \phi$ oscillations which one normally would associate with a $d$-wave symmetry, and $d$-wave gaps have constant (plus-minus) components which one would normally associate with an $s$-wave symmetry. When these "wrong" components are large, the gaps have accidental nodes. These nodes may be present or absent for both $s$-wave and $d$-wave gaps, i.e., symmetry constraints play no role here.

An $s$-wave gap with nodes in one of the "exotic" options offered by the electronic structure of FeSCs. Another "exotic" option is a $d$-wave state without nodes. In heavily electrondoped FeSCs, hole states are gapped, and only electron FSs remain. The $d$-wave gaps on these two FSs have no nodes if $\cos 2 \phi$ oscillation component is smaller than a constant term, hence the system will display a behavior typical for a fully gapped SC despite that the gap actually has a d-wave symmetry. There are even more exotic options offered by the actual three-dimensionality of the electronic structure and/or the hybridization of the electron FSs due to interaction via a pnictide/chalcogen. One RPA calculation [59] places the nodes of the $s^{ \pm}$gap on hole pockets, near particular $k_{z}$. Another study argues [58] that a $d$-wave gap for heavily electron doped FeSCs must have nodes near $k_{z}=\pi / 2$.

It is essential, however, that either a more conventional one or a more exotic pairing state develops only if the corresponding eigenvalue $\lambda_{i}$ is positive. To understand under what conditions $\lambda_{i}>0$ we now have to consider specific models for FeSCs. 


\section{THE INTERPLAY BETWEEN INTRA-POCKET AND INTER-POCKET IN- TERACTIONS}

In this section we make the first step in the analysis of what causes the attraction in FeSCs and consider how the sign and magnitude of $\lambda_{i}$ depends on the interplay between intra-pocket and inter-pocket interactions.

\section{A. Toy two-pocket model}

As a warm-up, consider first an idealized two-pocket model consisting of two identical circular pockets: a hole pocket at $(0,0)$ and an electron pocket at $(\pi, \pi)$. Because the electron FS is centered at $k$ along the diagonal, the $\cos 2 \phi$ terms in the electronic gap are no longer present, so some part of the physics of FeSCs is lost. Still, this idealized model is a good staring point to consider the interplay between intra-pocket and inter-pocket interactions.

Compared to Eqs. (9-13), we now have $\Gamma_{h h}^{s}=U_{h h}, \Gamma_{h e}^{s}=U_{h e}, \Gamma_{e e}^{s}=U_{e e}$, and $\Gamma_{h h}^{d}\left(k_{F}, p_{F}\right)=\tilde{U}_{h h} \cos 2 \phi_{k} \cos 2 \phi_{p}, \Gamma_{h e}^{d}(k, p)=\tilde{U}_{h e} \cos 2 \theta_{k}, \Gamma_{e e}^{s}=\tilde{U}_{e e}$.

The eigenvalue problem, Eq. (2), reduces to the set of two coupled equations in each channel. Solving them, we obtain

$$
\lambda_{1,2}^{s}=\frac{-\left(u_{h h}+u_{e e}\right) \pm \sqrt{\left(u_{h h}-u_{e e}\right)^{2}+4 u_{h e}^{2}}}{2}
$$

and

$$
\lambda_{1,2}^{d}=\frac{-\left(\tilde{u}_{h h}+\tilde{u}_{e e}\right) \pm \sqrt{\left(\tilde{u}_{h h}-\tilde{u}_{e e}\right)^{2}+4 u_{h e}^{2}}}{4}
$$

where $u_{i j}=U_{i j} N_{F}$ and $N_{F}=m /(2 \pi)$ is the density of states (DoS) per spin projection.

We see that a solution with a positive $\lambda$ in either $s$-or $d$-wave channel exists if the inter-pocket interaction is larger than intra-pocket interactions. Specifically, one needs

$$
u_{h e}^{2}>u_{e e} u_{h h} \text { or } \tilde{u}_{h e}^{2}>\tilde{u}_{e e} \tilde{u}_{h h} .
$$

If this condition is not met, the system remains in the normal state down to $T=0$. If Eq. (17) is satisfied and $u_{h e}>0, s$-wave solution with $\lambda^{s}>0$ yields a "plus-minus" gap, $\Delta_{e}=-\Delta_{h}\left(s^{ \pm}\right.$state). If $u_{h e}<0$ (what requires intra-band attraction), $s$-wave solution is a conventional one, with $\Delta_{e}=\Delta_{h}$. Similarly, $d$-wave solutions yield $\cos 2 \phi$ gaps on the hole and electron FSs with either zero or $\pi$ phase shift. 


\section{B. Multi-band models}

Consider next a more realistic case of two electron FSs centered at $(0, \pi)$ and $(\pi, 0)$. Now hole-electron and electron-electron interactions have $\cos 2 \phi$ terms, and the eigenvalue/eigenfunction problem, Eq. (2), reduces to the set of either four (or five) coupled equations in either s-wave or d-wave channels: two (or three) $\Delta$ 's are the gaps on the hole FSs, and two other $\Delta$ 's are angle-independent and $\cos 2 \phi$ components of the gaps on the electron FSs. Accordingly, there are either four or five different $\lambda_{s}$ and $\lambda_{d}$.

The analysis of $4 \times 4$ or $5 \times 5$ gap equations is tedious but straightforward. I will not discuss it in length (for a detailed discussion see Refs. [64,66]) but rather focus on an issue of whether it is still required that the inter-pocket interaction $u_{h e}$ exceeds the threshold set by intra-pocket hole-hole and electron-electron interactions. Interestingly enough, this may no longer be necessary. To illustrate this, consider the case of an $s$-wave pairing in a four-pocket model and assume for simplification that only one hole pocket is relevant to the pairing. Then the eigenvalue problem reduces to the set of three equations for $\Delta_{h}, \Delta_{e}$, and $\bar{\Delta}_{e}\left(\Delta_{e}(k)=\Delta_{e}+\bar{\Delta}_{e} \cos 2 \phi_{k}\right)$. Solving the set, we find three solutions $\lambda_{i}^{s}(i=1,2,3)$. In the absence of $\cos 2 \phi$ terms in $\Gamma_{i j}(k, p), \lambda_{3}^{s}=0$, and $\lambda_{1,2}^{s}$ are given by (15) with $u_{e e} \rightarrow 2 u_{e e}$ and $u_{h e}^{2} \rightarrow 2 u_{h e}^{2}$. Obviously, $u_{h e}$ has to exceed a threshold, otherwise $\lambda_{1,2}^{s}<0$. Once the angle dependent terms in (9-10) become non-zero, $\lambda_{3}^{s}$ also becomes non-zero, and its sign depends on the interplay between $\alpha_{h e}, \alpha_{e e}$, and $\beta_{e e}$. In particular, when $u_{h e}^{2}<u_{e e} u_{h h}$ (and, hence, $\left.\lambda_{1,2}<0\right), \lambda_{3}^{s}$ is positive or negative depending on whether or not $A>0$, where

$$
A=4 u_{e e} u_{h h}\left(\alpha_{e e}^{2}-\beta_{e e}\right)+u_{h e}^{2}\left(\alpha_{h e}^{2}+2 \beta_{e e}-3 \alpha_{h e} \alpha_{e e}\right)
$$

When the angle-dependence of the electron-electron interaction can be neglected, i.e., $\alpha_{e e}=$ $\beta_{e e}=0, \lambda_{3}^{s}>0$ no matter what is the ratio of $u_{h e}^{2}$ and $u_{e e} u_{h h}$. In particular, for $u_{h h} u_{e e}>u_{h e}^{2}$ and $\alpha_{h e}<<1$,

$$
\lambda_{3}^{s}=\alpha_{h e}^{2} \frac{2 u_{h e}^{2} u_{h h}}{u_{h h} u_{e e}-u_{h e}^{2}}>0
$$

In other words, for one of $s$-wave solutions, $\lambda^{s}>0$ even if intra-pocket repulsions are the largest. The full solution of the $3 \times 3$ set with $\alpha_{e e}=\beta_{e e}=0$ shows that two $\lambda$ 's are repulsive and one is attractive for arbitrary $u_{h e}^{2} / u_{e e} u_{h h}$. When the ratio is small, the attractive solution is close to (19), when the ratio is large, the attractive solution is close to $\lambda_{1}^{s}$ in (15). I illustrate this in Fig. 6 

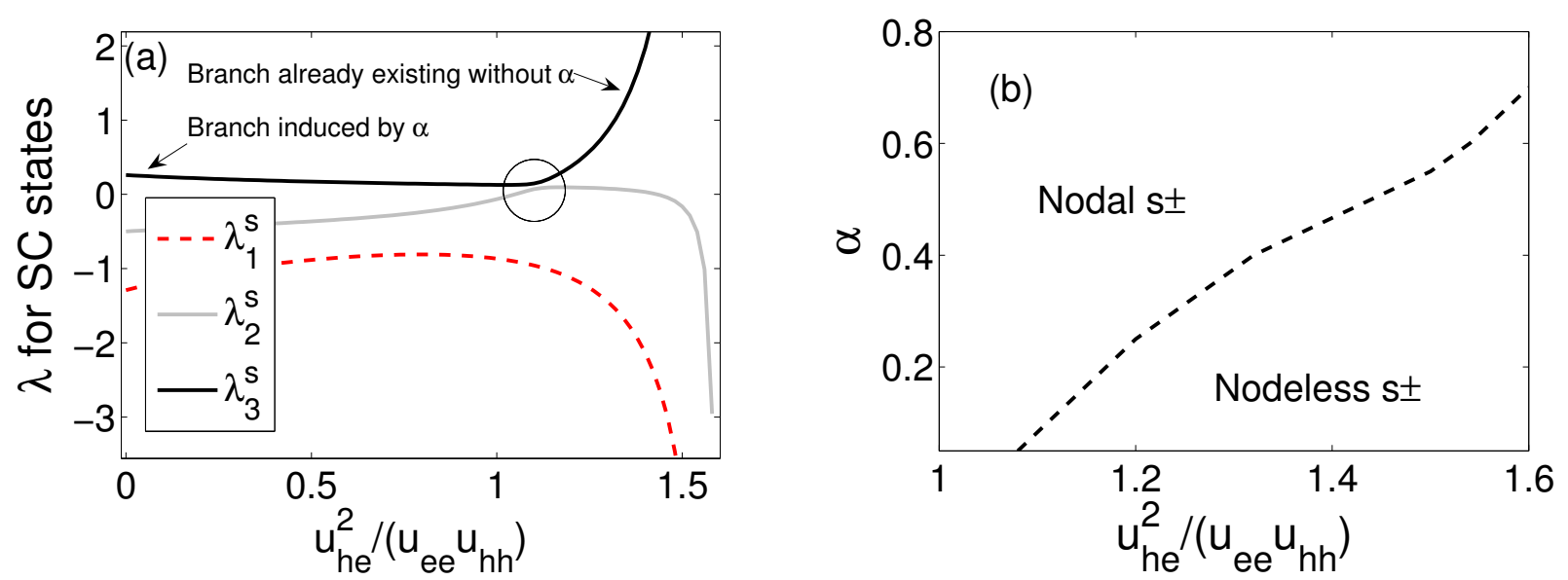

FIG. 6: (a) The three eigenvalues in the $s$-wave channel $\lambda_{i}^{s}$ as functions of $u_{h e}^{2} /\left(u_{e e} u_{h h}\right)$ for $\alpha_{e e}=\beta_{e e}=0$ and $\alpha_{h e}=0.4$. For any $u_{h e}^{2} /\left(u_{e e} u_{h h}\right)$, one $\lambda_{i}^{s}$ is positive (attractive), other two are negative. Positive $\lambda_{i}^{s}$ corresponds to $s \pm$ pairing. At small $u_{h e}^{2} /\left(u_{e e} u_{h h}\right)$ pairing is induced by $\alpha_{h e}$ and the gap has nodes on electron FSs. At large $u_{h e}^{2} /\left(u_{e e} u_{h h}\right)$ positive $\lambda_{i}^{s}$ exists already at $\alpha_{h e}=0$, and the gap along electron FS has nodes only if $\alpha_{h e}$ is above the threshold. The circle marks the area where positive and negative solutions come close to each other. The splitting between the two increases with $\alpha_{h e}$. (b) The regions of nodeless and nodal $s^{ \pm}$gap, depending on $\alpha_{h e}$ and $u_{h e}^{2} / u_{e e} u_{h h}$. From Ref. [64].

There is, however, one essential difference between the cases $u_{h e}^{2} / u_{e e} u_{h h}>1$ and $u_{h e}^{2} / u_{e e} u_{h h}<1$. In the first case, momentum-dependence of the interaction just modifies the "plus-minus" solution which already existed for momentum-independent interaction. In this situation, the gap along electron FS gradually acquires some $\cos 2 \phi$ variation and remains nodeless for small $\alpha_{h e}$. In the second case, the solution with $\lambda>0$ is induced by the momentum dependence of the interaction, and the eigenvalue corresponding to $\lambda_{3}^{s}$ necessary has $\bar{\Delta}_{e}>\Delta_{e}$, i.e., $s$-wave gap has nodes along the electron FS [67]. In other words, the pairing occurs for all parameters but whether the gap is nodal or not at small $\alpha_{h e}$ depends on the relative strength of intra-pocket and inter-pocket interactions. When intra-pocket interaction dominates, the gap "adjusts" and develops strong $\cos 2 \phi$ component which does not couple to a momentum-independent $u_{e e}$ term and by this effectively reduces the strength of electron-electron repulsion.

The same reasoning holds for the case of two non-equivalent hole FSs, and for 5-pocket models, and also for the $d$-wave channel, For all cases, the solution with $\lambda_{i}>0$ may exist 

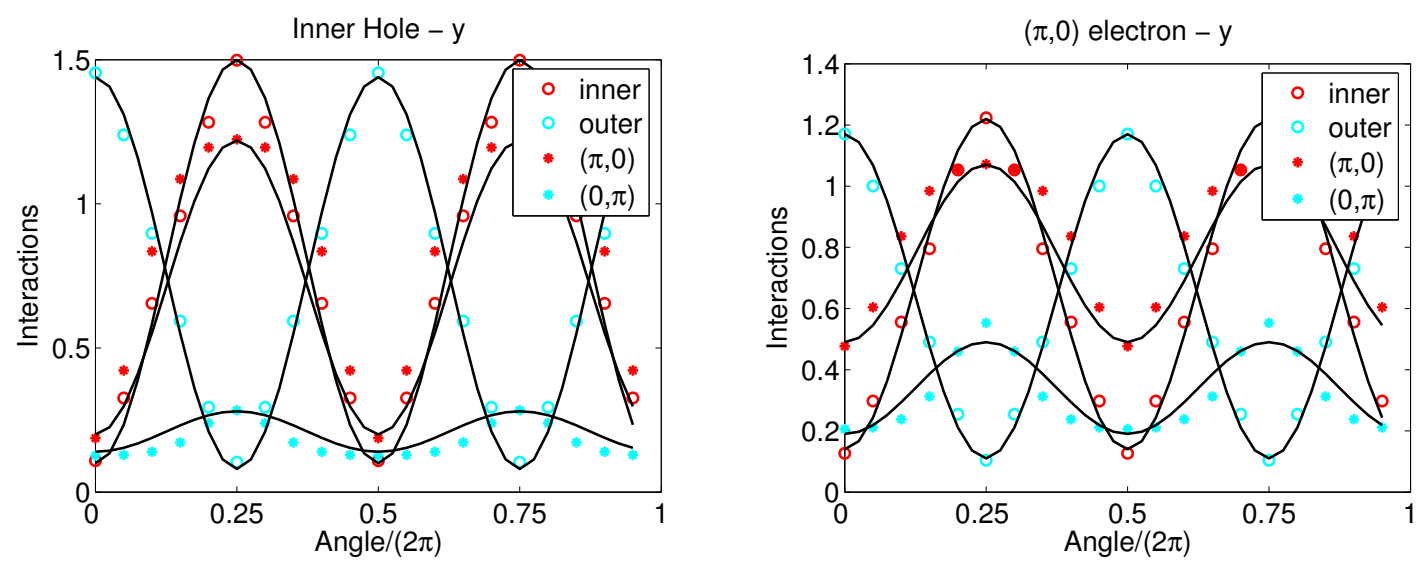

FIG. 7: Representative fits of the interactions $\Gamma_{i j}\left(\mathbf{k}_{F}, \mathbf{p}_{F}\right)$ by LAHA for the 4-pocket model. $\Gamma_{i j}$ are obtained by converting the Hamiltonian, Eqs. (20), (21) from the orbital to the band basis. The symbols represent interactions computed numerically for the 5-band orbital model using LDA band structure, the black lines are the fits using Eqs. (9)-(13). The fit is for the set $U=1.67$, $J=J^{\prime}=0.21, V=1.46$, and $\mu=0.08$ (all in $\mathrm{eV}$ ). A positive $\mu$ corresponds to electron doping. $\mathbf{k}_{F}$ in $\Gamma_{i j}\left(\mathbf{k}_{F}, \mathbf{p}_{F}\right)$ is selected along $y$ direction on either an electron or a hole FS (its location is specified on top of each figure), and $\mathbf{p}_{F}$ is varied along each of FSs. The angle $\phi$ is measured relative to $k_{x}$.

even when intra-pocket interactions are the largest, but in this situation the gaps on the hole FSs have accidental nodes. The existence or non-existence of the solution at strong intra-pocket repulsion depends on the complex interplay between the prefactors of $\cos 2 \theta$ terms in electron-hole and electron-electron pairing vertices, see Eq. (18).

\begin{tabular}{|c|c|c|c|c|c|c|c|c|c|c|}
\hline & $u_{h_{1} h_{1}}$ & $u_{h_{2} h_{2}}$ & $u_{h_{1} h_{2}}$ & $u_{h_{1} e}$ & $\alpha_{h_{1} e}$ & $u_{h_{2} e}$ & $\alpha_{h_{2} e}$ & $u_{e e}$ & $\alpha_{e e}$ & $\beta_{e e}$ \\
\hline $\mathrm{NSF}$ & 0.8 & 0.76 & 0.78 & 0.46 & -0.24 & 0.4 & -0.30 & 0.77 & 0.14 & 0.09 \\
\hline $\mathrm{SF}$ & 2.27 & 2.13 & 2.22 & 4.65 & -0.34 & 2.29 & -0.22 & 3.67 & 0.15 & 0.04 \\
\hline & $\tilde{u}_{h_{1} h_{1}}$ & $\tilde{u}_{h_{2} h_{2}}$ & $\tilde{u}_{h_{1} h_{2}}$ & $\tilde{u}_{h_{1} e}$ & $\tilde{\alpha}_{h_{1} e}$ & $\tilde{u}_{h_{2} e}$ & $\tilde{\alpha}_{h_{2} e}$ & $\tilde{u}_{e e}$ & $\tilde{\alpha}_{e e}$ & $\tilde{\beta}_{e e}$ \\
\hline $\mathrm{NSF}$ & 0.7 & 0.66 & -0.68 & -0.25 & -0.58 & 0.24 & -0.42 & 0.11 & -0.5 & 0.25 \\
\hline $\mathrm{SF}$ & 1.50 & 1.40 & -1.50 & -3.73 & -0.44 & 1.44 & -0.32 & 1.03 & -0.49 & -0.02 \\
\hline
\end{tabular}

TABLE I: Table for $s$-wave and $d$-wave parameters for the same set as in Fig. 7 . NSF and SF mean the bare interaction without the spin-fluctuation component and the full interaction, respectively. 


\section{How to extract $\Gamma_{i j}(\mathbf{k}, \mathbf{p})$ from the orbital model?}

So far, in our discussion $u_{i j}, \alpha_{i j}$, etc, are treated as some phenomenological inputs. To obtain the actual values of these parameters, one needs a microscopic model. The most commonly considered model for FeSCs is an effective 5-orbital model for $F e$ atoms with local intra-orbital and inter-orbital hopping integrals and intra-orbital and inter-orbital densitydensity (Coulomb) repulsions, Hund-rule exchange, and the pair hopping term.

$$
H_{i n t}=\sum_{i s} U_{i i} n_{i, s \uparrow} n_{i s \downarrow}+\sum_{i, s, t \neq s} \frac{V_{s t}}{2} n_{i s} n_{i t}-\sum_{i, s, t \neq s} \frac{J_{s t}}{2} \vec{S}_{i s} \cdot \vec{S}_{i t}+\frac{1}{2} \sum_{i, s, t \neq s} J_{s t}^{\prime} \sum_{\sigma} c_{i s \sigma}^{\dagger} c_{i s \bar{\sigma}}^{\dagger} c_{i t \bar{\sigma}} c_{i t \sigma}
$$

where $n_{i s}=n_{i, s \uparrow}+n_{i s \downarrow}$.

The Hamiltonian $H_{\text {int }}$ can be equivalently re-expressed via spin-independent interactions, as

$H_{i n t}=\sum_{i s} U_{i i} n_{i, s \uparrow} n_{i s \downarrow}+\sum_{i, s, t \neq s} \frac{\bar{U}_{s t}}{2} n_{i s} n_{i t}+\sum_{i, s, t \neq s} \frac{J_{s t}}{4} c_{i s \sigma}^{\dagger} c_{i t \sigma} c_{i t \bar{\sigma}}^{\dagger} c_{i t \bar{\sigma}}+\frac{1}{2} \sum_{i, s, t \neq s} J_{s t}^{\prime} \sum_{\sigma} c_{i s \sigma}^{\dagger} c_{i s \bar{\sigma}}^{\dagger} c_{i t \bar{\sigma}} c_{i t \sigma}$

where $\bar{U}_{s t}=V_{s t}+J_{s t} / 4$.

The hopping integrals (36 total) are obtained from the fit to DFT band structure. [69] For the interaction parameters, the most common approximation is to assume that $\bar{U}_{s t}, J_{s t}$ and $J_{s t}^{\prime}$ are independent of the orbital indices $s$ and $t$, as long as $s \neq t$. The model can be also extended to include non-local Fe-Fe interactions via a pnictide [68].

The bare parameters in (20) and (21) are inter-related due to local spin-rotation invariance $[19,35]$, but that invariance is broken if we view (20) and (21) as an effective low-energy model in which the interactions are dressed by the renormalizations coming from fermions with energies of order bandwidth. By this reason, in most studies $U, \bar{U}, J$, and $J^{\prime}$ are treated as independent parameters.

We now need to convert (20), (21) into the band basis and re-express it in the form of Eq. (1). This is done by transforming into the momentum space, introducing new, hybridized operators, which diagonalize the quadratic form, and re-expressing the interaction terms in (20) or (21) in terms of these new operators. The end result of this procedure is the effective 
Hamiltonian in the band basis which has the form of Eq. (1) with $\Gamma_{i j}(\mathbf{k}, \mathbf{p})$ given by

$$
\begin{aligned}
\Gamma_{i j}\left(\mathbf{k}, \mathbf{k}^{\prime}\right)= & \sum_{s t p q} \alpha_{i}^{t, *}(-\mathbf{k}) \alpha_{i}^{s, *}(\mathbf{k}) \operatorname{Re}\left[\Gamma_{s t}^{p q}\left(\mathbf{k}, \mathbf{k}^{\prime}\right)\right] \\
& \times \alpha_{j}^{p}\left(\mathbf{k}^{\prime}\right) \alpha_{j}^{q}\left(-\mathbf{k}^{\prime}\right),
\end{aligned}
$$

where $\left[\Gamma_{s t}^{p q}\left(\mathbf{k}, \mathbf{k}^{\prime}\right)\right.$ are linear combinations of $U, \bar{U}, J$ and $\bar{J}$, and $\alpha_{i}^{p}$ is the matrix element connecting the original fermionic operator $c_{p}$ in the orbital basis with the new fermionic operator $a_{i}$ on FS $i$ in the band basis. The matrix elements $\alpha_{i}^{p}$ contain information which orbitals mostly contribute to a particular segment of a particular FS [19,20]. Because of this, the interaction $\Gamma_{i j}(\mathbf{k}, \mathbf{p})$ in the band basis generally depends on the angles along different FSs and contains components in all representations of the tetragonal $D_{4 h}$ group.

The angle dependence of $s$-wave and $d_{x^{2}-y^{2}}$ vertices agrees by symmetry with Eqs (9)(13). What s a'priori unknown is how well the interactions can be approximated by the leading angle harmonics, i.e., whether the terms labeled as ... in (9)-(13) can actually be neglected. This issue was analyzed in detail in Ref. [66], and the answer is affirmative - the LAHA works rather well. In Fig.7 I show representative fits for a particular set of parameters and in Table $1 \mathrm{I}$ show $u_{e h}$ and other parameters, extracted from the fit (in the lines marked marked "NSF", meaning that this is for the bare interaction, without extra spin-fluctuation component (see below). The results somewhat vary depending on the values of $U, V, J, J^{\prime}$, but in general intra-band interactions in the $s$-wave channel, $u_{e e}$ and $u_{h h}$, exceed interband $u_{h e}$. This is not surprising because $u_{e e}$ and $u_{h h}$ are essentially Coulomb interactions at small momentum transfers, while $u_{e h}$ is the interaction at large momentum transfer, and it should be smaller on general grounds. Only when $V=J=J^{\prime}=0$, the interaction in the band basis becomes independent on the momentum [70], i.e., $u_{e e}=u_{h h}=u_{h e}$ (this was termed "Coulomb avoidance" in Ref. [23]). According to Table I, intra-band interactions are also larger in the d-wave channel: $\tilde{u}_{h_{i} h_{i}} \tilde{u}_{e e}>\tilde{u}_{h_{i} e}^{2}$, although the reasons why this is the case are not transparent.

According to the analysis in Sec.II, as long as intra-orbital interactions exceed interorbital interaction, $\lambda_{i}$ are negative (repulsive) if we neglect $\cos 2 \phi$ terms in $\Gamma_{i j}$. If we don't neglect these terms, $\lambda_{i}$ can be positive even for small $u_{h e}$ or $\tilde{u}_{h e}$ but this requires that angledependent term satisfy the inequality in Eq. (18). The $\alpha_{e h}, \alpha_{e e}$, and $\beta_{e e}$, and their $d$-wave analogs in Table I are such that this condition is not me, that is for the bare interaction neither $s$-wave nor $d$-wave pairing is possible. 
The conditions on $\alpha, \beta$, etc depend on the parameters used for the hopings. Varying these parameters (e.g., making $J$ larger) one can, in principle, met the condition in Eq. (18) and obtain either $s$-wave or $d$-wave solution with a positive $\lambda_{i}$ and with strong $\cos 2 \phi$ oscillations of the gap along electron FSs. But the generic trend is that the bare interaction yields repulsive $\lambda_{i}$ in $s$-wave and $d$-wave channels.

\section{HOW TO OVERCOME INTRA-POCKET REPULSION}

How to overcome strong intra-pocket repulsion is the major issue for FeSCs. A conventional McMillan-Tolmachev renormalization [71] that reduces Coulomb repulsion and allows electron-phonon attraction to overcome it does not help at this stage because both $u_{e e} u_{h h}$ and $u_{h e}^{2}$ renormalize in the same way, and if repulsive $u_{e e} u_{h h}$ part is initially stronger, the renormalization just reduces the strength of the total repulsive interaction, but doesn't change its sign.

The generic idea how to overcome Coulomb repulsion goes back to Kohn-Luttinger approach to superconductivity [32] The pairing interaction I considered so far is the bare interaction between a particular pair of fermions. The actual pairing interaction $\Gamma_{i j}(\mathbf{k}, \mathbf{p})$ is given by the fully renormalized irreducible vertex in the particle-particle channel. This irre-

ducible vertex is the sum of the original (bare) interaction and terms of second and higher order in $U, V$, etc. Part of these higher-order terms account for the screening of the original interaction by particle-hole pairs, another part can be re-cast as effective interactions mediated by collective bosonic degrees of freedom in either charge or spin channel. The issue I discuss now is how to obtain dressed pairing vertex.

\section{A. RPA approach}

One route is to adopt a semi-phenomenological approach and assume that the system is reasonably close to a particular density-wave ordered phase, such that the strongest component of the effective interaction is mediated by near-gapless fluctuations of this particular density-wave order. The approach goes back to Berk and Schrieffer [74], who considered effective pairing interaction mediated by ferromagnetic spin fluctuations. In recent years it has been applied to cuprates [75] and other correlated electron materials [76]. Most of 

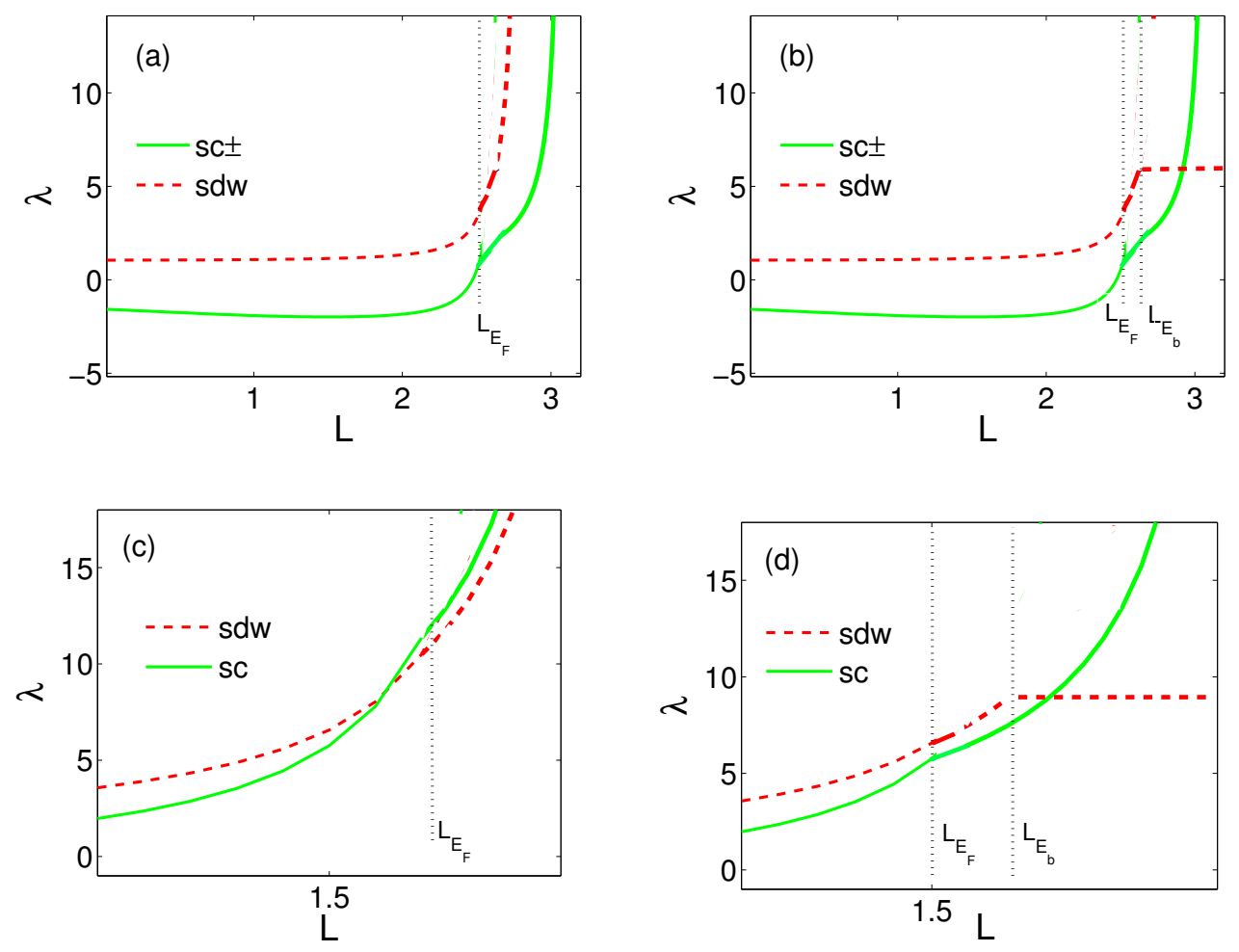

FIG. 8: Running vertices in SDW and SC $s \pm$ channels for the 2-pocket model, panels (a)-(b), and the 4-pocket model, panels (c)-(d), as functions of the RG parameter $L=\log W / E$. For the 2 pocket model $\lambda^{S D W}$ is the largest along the whole RG flow at perfect nesting (a), but $\lambda^{s^{ \pm}}$ eventually wins at a finite doping when $\lambda^{S D W}$ is cut below some $E_{b}$. For the 4-pocket model, $\lambda^{s^{ \pm}}$ can be the largest coupling already at perfect nesting (c), or exceed $\lambda^{S D W}$ above some critical doping (d). Figures are from Ref. [64].

parent compounds of FeSCs possess long-range SDW order, and it is natural to assume that magnetically mediated pairing interaction plays the central role.

There is no rigorously justified diagrammatic derivation of an effective fermion-fermion interaction mediated by collective modes, except for special cases [76]. The pragmatic approach is to select a set of ladder-type or bubble-type diagrams which give rise to a SDW order above some interaction threshold and use the same set of diagrams below the threshold. This approach is termed RPA by analogy with the screening problem. A generic way to apply RPA is to antisymmetrize the original interaction, decompose it into spin and charge channels and renormalize each interaction in a ladder/bubble approximation, neglecting cross-terms. Each 4-fermion interaction component $\Gamma_{s t}^{p q}$ then contains two combinations of fermionic spin 
indices: $\delta_{\alpha, \beta} \delta_{\gamma, \delta}$ and $\vec{\sigma}_{\alpha \beta} \vec{\sigma}_{\gamma \delta}$, where $\sigma$ are Pauli matrices. These two combinations have to be convoluted with the spin structure $i \sigma^{y}$ of the two-particle anomalous pairing vertex. Using $\sigma_{\alpha \gamma}^{y} \delta_{\alpha, \beta} \delta_{\gamma, \delta}=\sigma_{\beta \delta}^{y}$ and $\sigma_{\alpha \gamma}^{y} \sigma_{\alpha \beta} \sigma_{\gamma \delta}=-3 \sigma_{\beta \delta}^{y}$ and incorporating the factor -3 into the spin part of the effective pairing vertex, one obtains, in the orbital basis

$$
\begin{aligned}
\Gamma_{s t}^{p q}(\mathbf{k}, \mathbf{p}) & =\left[\frac{1}{4} \Gamma^{s}+\frac{3}{4} \Gamma^{c}+\frac{3}{2}\left(\Gamma^{s}\right)^{2} \chi_{s}^{R P A}(\mathbf{k}-\mathbf{p})\right. \\
& \left.-\frac{1}{2}\left(\Gamma^{c}\right)^{2} \chi_{c}^{R P A}(\mathbf{k}-\mathbf{p})\right]_{p s}^{t q}
\end{aligned}
$$

where $\Gamma^{s}$ and $\Gamma^{c}$ are spin and charge components of the bare antisymmetrized interaction, $\chi_{s}^{R P A}$ is the dressed static spin susceptibility and $\chi_{c}^{R P A}$ is the dressed static charge susceptibility. The interaction, Eq. (23), is then converted into the band basis using (22), and the pairing in different channels is analyzed in the same way as it was done for the bare interaction, i.e., either by explicitly solving integral equation on $\lambda_{i}$ in a particular channel [19-21,77,81], or by approximating $\Gamma_{i j}(\mathbf{k}, \mathbf{p})$ by the leading angular harmonics and solving $4 \times 4$ or $5 \times 5$ gap equations (see $\mathrm{SecV}$ below). The full analysis of the pairing problem often requires one to know the frequency dependence of $\chi$, but for the analysis of the pairing symmetry and the momentum dependence of the gap the frequency dependence is not overly relevant and I neglect it.

In all model calculations, the spin susceptibility $\chi_{s}^{R P A}$ is enhanced due to close proximity to SDW order, the charge susceptibility $\chi_{c}^{R P A}$ remains small and can be neglected. How $\left(\Gamma^{s}\right)^{2} \chi_{s}^{R P A}$ in (23) modifies $u_{i j}$ depends on the type of SDW order. For moderately doped FeSCs $\chi_{s}^{R P A}(q)$ is peaked at $(0, \pi)$ and $(\pi, 0)$, which are the two momenta separating hole and electron FSs. Not surprisingly, electron-hole interaction $u_{h e}$ is enhanced relative to $u_{e e}$ and $u_{h h}$. By less obvious reasons, d-wave component of electron-hole interaction $\tilde{u}_{h e}$ is also enhanced, although not as strongly as $u_{h e}$. As an illustration, I show in Table I, in the lines marked as SF, renormalized interactions for the same set of input parameters which were considered in the previous section. Clearly, $u_{h e}$ and $\tilde{u}_{h e}$ are enhanced. If the enhancement is strong enough, either Eq. (18) becomes valid, or $u_{h e}^{2} / u_{e e} u_{h h}$ becomes larger than one (or $\tilde{u}_{h e}^{2} / \tilde{u}_{e e} \tilde{u}_{h h}$ becomes larger than one), and the system becomes unstable towards s-wave or d-wave superconductivity, whichever $\lambda_{i}$ is the largest.

This scenario requires bare $\Gamma^{s}$ to be positive. Only then spin susceptibility $\chi_{s}^{R P A}(q)$ is enhanced. In the orbital basis, this requirement essentially means that intra-orbital Coulomb repulsion $U$ must be larger than inter-orbital $\bar{U}$. In the band basis, the same requirement 


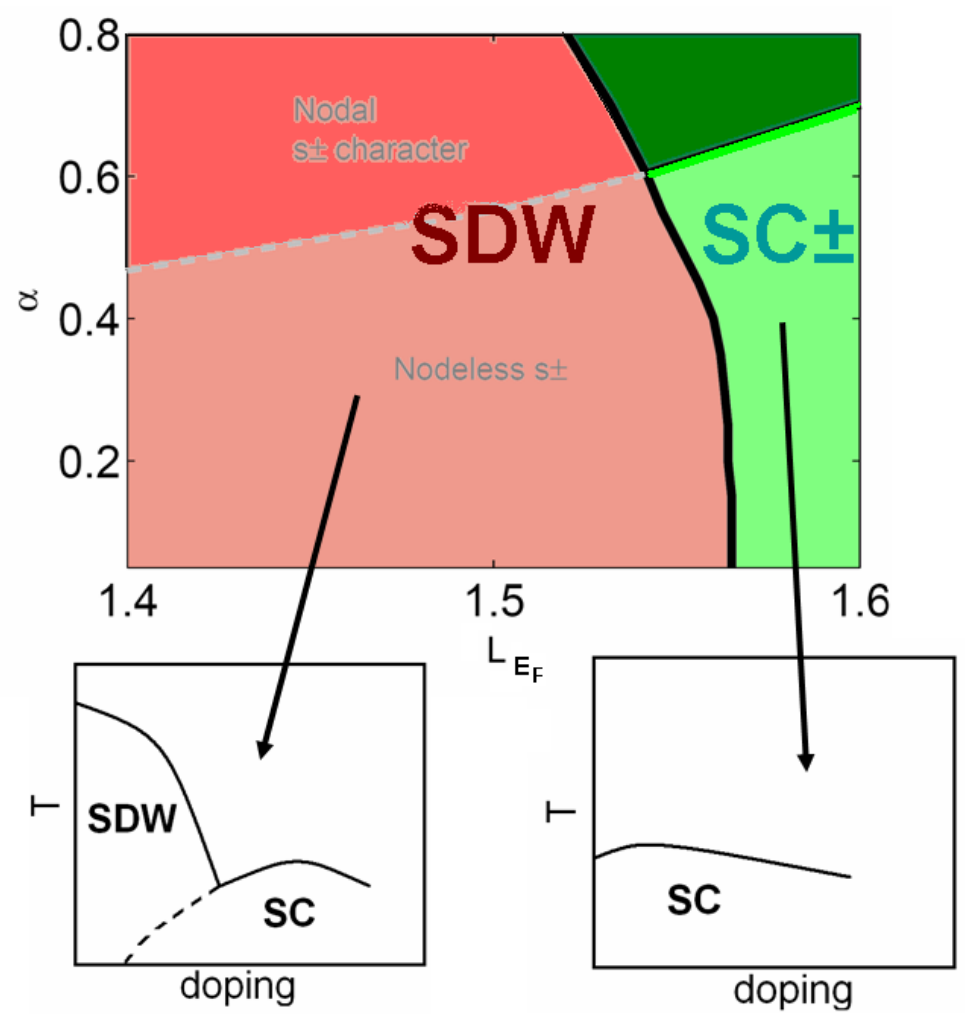

FIG. 9: Top panel: the phase diagram of the 4-pocket model at zero doping as the function of the strength of the angle-dependent component of the interaction, $\alpha$, and $L_{E_{F}}=\log W / E_{F}$, where $W$ is the bandwidth and $E_{F}$ is the Fermi energy. Lower panels - the phase diagrams as functions of doping for different $L_{E_{F}}$. The superconducting gap in $s^{ \pm}$state may be nodeless or have nodes along electron FSs. In the region labeled as SDW, SC emerges only at a finite doping, when SDW instability is cut. In the region labeled as $S C^{ \pm}, \mathrm{SC}$ state emerges already at zero doping. Figure is from Ref. [64].

implies that bare $u_{h e}$ must be positive [70]. What if bare $u_{h e}$ is negative? Then $\left|u_{h e}\right|$ may still get an enhancement within RPA, but the enhancement now comes from charge (orbital) fluctuations [36]. The latter are strong if the system is close to a state with an orbital order. There are proposals [37] that broken rotational invariance observed above $T_{N}$ in most of FeSCs [78] may be due to orbital order. [An alternative scenario [61] is an Ising order associated with $Z_{2}$ degree of freedom separating $(0, \pi)$ and $(\pi, 0)$ spin configurations.] If $u_{h e}^{2}$ is enhanced by orbital fluctuations such that it again exceeds $u_{e e} u_{h h}$, s-wave SC again develops, but the eigenvalue now corresponds to a conventional $s^{++}$gap, with the same sign 
along hole and electron FSs [36]. I will compare experimental evidence for $s^{ \pm}$vs $s^{++}$gap in Sec.VI. For the rest of this section I assume that $u_{h e}>0$, i.e., the attractions is due to spin fluctuations.

There are two underlying assumptions in the RPA approach. The obvious one is the selection of only ladder or bubble diagrams in the absence of a small parameter. The less obvious assumption is the very idea that the pairing can be viewed as mediated by collective density-wave bosonic excitations, because "mediation" implies that density-wave fluctuations develop at energies well above relevant energies for the pairing. This last idea is reasonably well justified for the cuprates, in which magnetic fluctuations develop at energies of a few hundred med, well above $T_{c}$. Whether this is also the case for FeSCs is less obvious as magnetism in most of these materials is itinerant (parent compounds are metals), and the highest $T_{c}$ is at dopings at which SC and SDW ordering temperatures almost coincide. Whether in this situation spin fluctuations can be viewed as "pre-existing" for the $\mathrm{SC}$ problem is not obvious. It is then instructive to consider also an alternative approach which treats magnetic and SC fluctuations on equal footings. This alternative approach is based on RG technique and is termed as "RG"

\section{B. RG approach}

RG approach is unbiased in the sense that it doesn't assume that the pairing is mediated by a collecting bosonic degree of freedom. Rather, it departs from a bare Hamiltonian with the original 4-fermion interaction and studies how particle-particle and particle-hole susceptibilities evolve as one progressively integrates out contributions from fermions with energies larger than some running scale $E$. This should, in principle, address the issue whether spin fluctuation develop at a larger $E$ than pairing fluctuations. In practice, this approach is indeed also an approximate one because even at weak coupling one cannot explicitly sum up contributions from fermions with energies between the bandwidth $W$ and $E$. This can be done rigorously only if the renormalizations depend on the running scale logarithmically, i.e., are the functions of $L=\log W / E$. Then the flow of the couplings can be described by a set of differential equations $d u_{i j} / d L=f\left(u_{l m}\right)$, where $u_{l m}$ in $f\left(u_{l m}\right)$ are renormalized interactions at the same running scale L.

Renormalizations in a superconducting channel are indeed depend on $L$, but In this 
respect FeSCs are "gifts from nature" because density-wave renormalizations involving fermions near hole and electron FSs also scale as $\log W / E$ at $E$ above the threshold set by non-perfect nesting [70,79]. Then one can obtain a set of coupled RG equations for pairing and density-wave vertices. This set is called "parquet" because the renormalizations in the particle-particle channel are often represented as horizontal ladder diagrams, renormalizations in the particle-hole (density-wave) channel as vertical ladder diagrams, and taking both on equal footing amounts to building perturbatively a parquet pattern.

From physics perspective, the key feature of RG approach is that the pairing, SDW, and CDW fluctuations all "talk to each other" at intermediate energies. Not only pairing fluctuations are enhanced by SDW fluctuations, but also SDW fluctuations are affected by pairing fluctuations and so on.

To get the idea how the RG works in FeSCs, consider momentarily a toy two-pocket model. Previously, in Sec. III A, I introduced three interactions which contribute to the pairing: intra-pocket hole-hole and electron-electron interactions $u_{h h}$ and $u_{e e}$ and inter-pocket holeelectron interaction $u_{h e}>0$. Suppose that bare electron-hole interaction is weak and the bare $\lambda^{s^{ \pm}}<0$. Now let's add two other interactions which do not directly contribute to the pairing but contribute to density-wave instability: density-density and exchange interactions between fermions from hole and electron pockets, $u_{d d}$ and $u_{e x}$, respectively. The coupling in the SDW channel is $\lambda^{S D W}=u_{d d}+u_{h e}$. As one progressively integrates out contributions from high energies, all couplings evolve and obey

$$
\begin{aligned}
& \dot{u}_{d d}=u_{d d}^{2}+u_{h e}^{2} \\
& \dot{u}_{e x}=2 u_{e x}\left(u_{d d}-u_{e x}\right) \\
& \dot{u}_{h e}=u_{h e}\left(4 u_{d d}-2 u_{e x}-u_{h h}-u_{e e}\right) \\
& \dot{u}_{h h}=-u_{h e}^{2}-u_{h h}^{2} \\
& \dot{u}_{e e}=-u_{h e}^{2}-u_{e e}^{2}
\end{aligned}
$$

where the derivatives are with respect to $\bar{L}=(1 / 2) \log W / E$. We see that $u_{h e}$ gets a boost from $u_{d d}$, which is the part of the SDW vertex, and in turn $u_{d d}$ gets a boost from $u_{h e}$. Solving the set, we find that $u_{h e}$ grows under RG while $u_{h h}$ and $u_{e e}$ decrease (and eventually change sign), such that below some $E_{0}$ the running $\lambda^{s^{ \pm}}$changes sign and becomes attractive.

The physics behind the sign change of $\lambda^{s^{ \pm}}$is essentially the same as in RPA analysis $u_{e h}$ gets a boost from SDW fluctuations. But RG analysis also addresses the issue of the 
interplay between SDW and SC orders as both are determined by the running interactions which "push" each other. For the two-pocket model SDW eigenvalue $\lambda^{S D W}$ remains larger than $\lambda^{s^{ \pm}}$along the whole RG trajectory (panels a and b in Fig. 8), but in multi-pocket systems more complex behavior is possible [64,80] (see panels c and d in Fig. 8 and Fig. $9)$.

The ability to address in an unbiased way what kind of order develops first is an obvious advantage of the RG approach. At the same same time, parquet RG has only limited applicability range because Eqs. (24) and the analogous equations for multi-pocket models [64] are valid only when the running $E$ is larger than the Fermi energy $E_{F}$. This wouldn't be an obstacle if the leading instability in FeSCs would develop already at $E>E_{F}$ (Refs. [70,72,73]). But this is unlikely given that $E_{F} \sim 10^{2} \mathrm{meV}$ is order of magnitude larger than $T_{N}$ and $T_{c}$. One then has to extend the analysis to $E<E_{F}$. It turns out that at such $E$ renormalizations in the SDW and SC channels decouple from each other [22,64] and each $\lambda^{i}$ evolves according to $d \lambda_{R}^{i} / d L=\left(\lambda_{R}^{i}\right)^{2}$ (where now $L=\log W / E$ ) Then SDW or SC instability occurs first depending on which $\lambda$ (either $\lambda^{S D W}$ or $\lambda^{s^{ \pm}}$) is larger at $E_{F}$. Away from a perfect nesting, the logarithmical RG flow of $\lambda^{S D W}$ is cut below some scale $E_{b}$, while $\lambda^{s^{ \pm}}$continue to grow and definitely becomes the leading instability above a certain doping. I illustrate this in Fig. 9.

This consideration shows that what parquet RG actually provides are the values of the dressed interactions $u_{i j}$ at the scale of $E_{F}$. Below this scale, SDW order develops without input from SC channel, and on the other hand SC develops in a BCS-fashion. If the doping is such that SC instability is the leading instability in the problem, the symmetry and the structure of the SC gap is analyzed in the same way as in Sec. III), only $u_{i j}$ and $\tilde{u}_{i j}$ are now dressed interactions, renormalized by the RG flow between $W$ and $E_{F}$. From this perspective, RPA and RG approaches are similar in the sense that the end result of both approaches is the effective BCS Hamiltonian at $E_{F}$ with renormalized interactions some of which are pushed up by SDW fluctuations.

There is another issue with RG related to angle-dependencies of the interactions (see Sec. III C). A conventional logarithmical RG is applicable only when $\alpha$ and $\beta$ terms in (9)-(13) are small. To order $O(\alpha), O(\beta)$, angle dependencies remains intact, only the overall factors $u_{i j}$ flow [64]. Beyond this order, logarithmical and non-logarithmical terms mix up and the selection of diagrams can no longer be rigorously justified. One way to proceed in this 

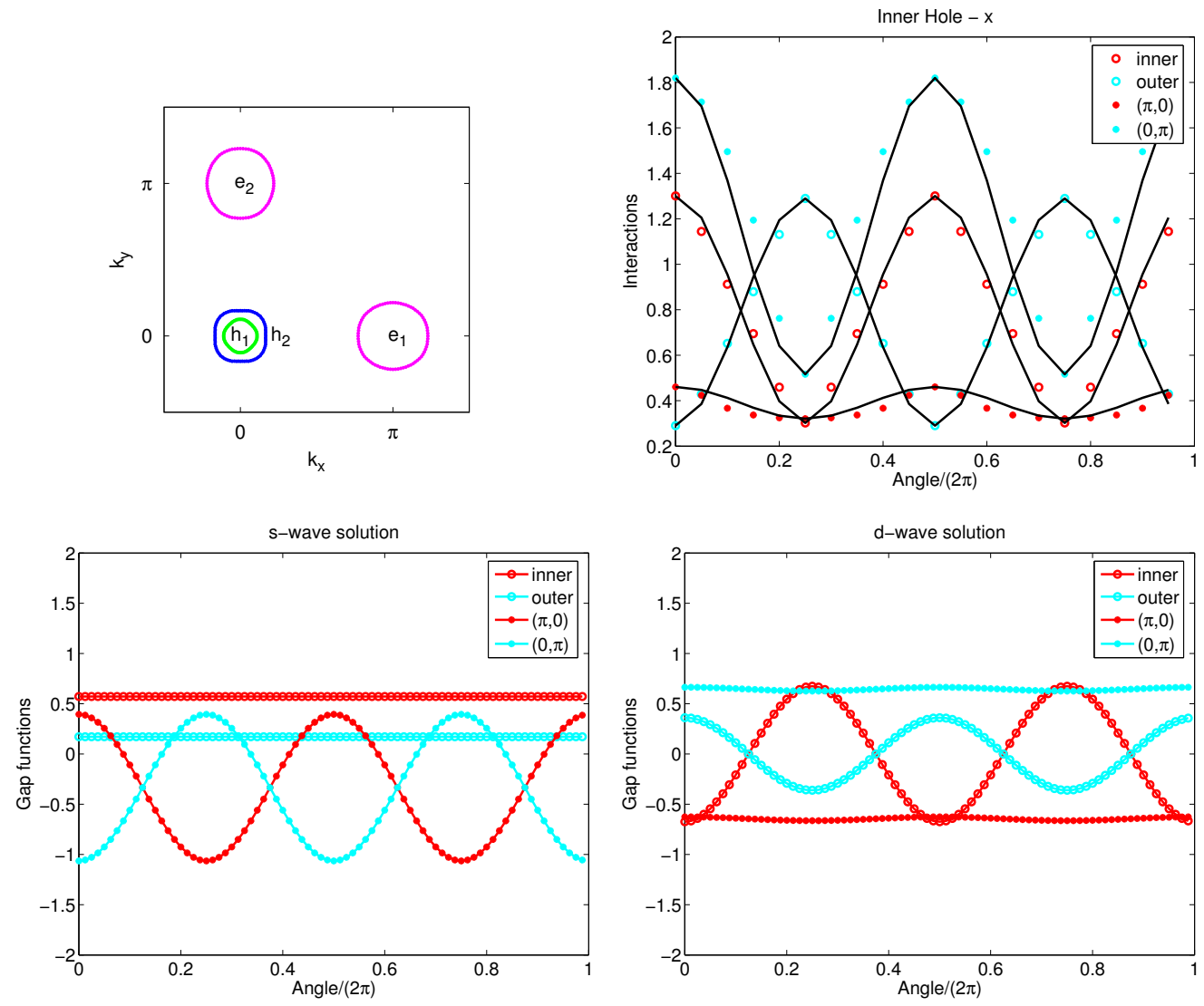

FIG. 10: Representative case of small/moderate electron doping, when both hole and electron pockets are present. Panel a - the FS, panel $\mathrm{b}$ - representative fits of the interactions by LAHA (the dots are RPA results, the lines are LAHA expressions, Eqs (9)-(13)). Panels c and d - the eigenfunctions in $s$-wave and $d$-wave channels for the largest $\lambda^{s}$ and $\lambda^{d}$. From Ref. [66].

situation is to keep the angle dependence intact by hand and use the same equations for $u_{i j}$ as if the interactions were angle-independent [64]. Another way is to partition each FS into patches and write a larger set of RG equations for the interactions between different patches. This last approach is called functional $\mathrm{RG}$ (fRG) $[65,80,86,87]$. fRG is not an exact approach as it mixes logarithmical and non-logarithmical terms, but it is nevertheless a very powerful numerical technique to analyze the interplay between density-wave and superconducting instabilities in systems with angle-dependent interactions.

Until now, the results of RPA, fRG and logarithmical RG fully agree with each other on (i) the interplay between SDW and SC instabilities, (ii) the symmetry of the SC state at different dopings, and (iii) the structure of the SC gap. This is a positive news because it likely implies that the underlying physics of FeSCs is quite robust. In particular, both 

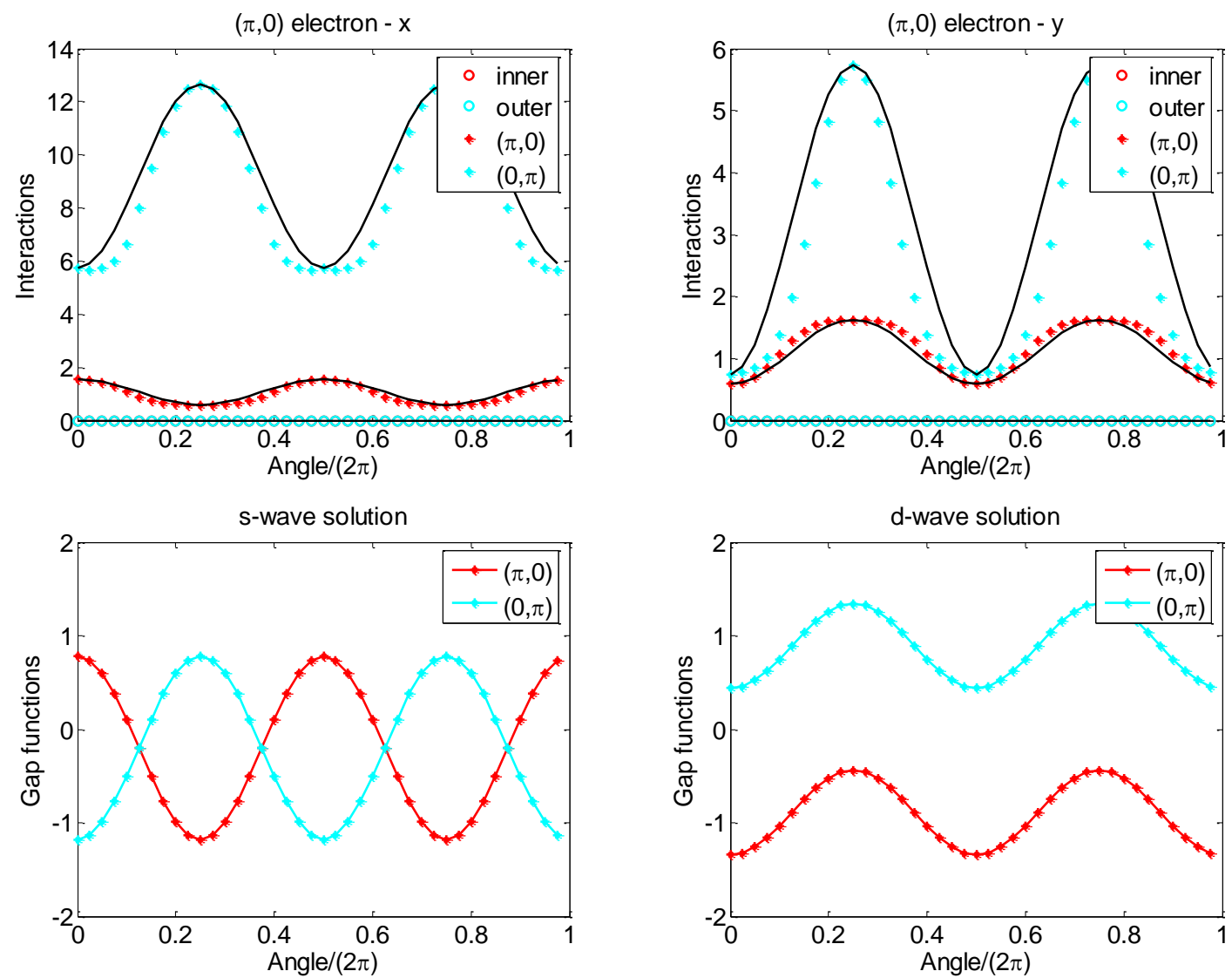

FIG. 11: The fits of the RPA interactions by LAHA and the structure of $s$-wave and $d$-wave gaps for the case of heavy electron doping, when only electron FSs are present. From Ref. [66].

fRG [80] and conventional RG [64] show that in 4-band systems SC may be the leading instability even without doping, while in 2-band and 5-band systems SDW is the leading instability at zero doping, and SC emerges only upon doping. RPA, fRG, and conventional RG all show that $s^{ \pm}$gap has nodes along electron FS in a much wider parameter range in 4-pocket systems than in 5-pocket systems because the additional hole FS at $(\pi, \pi)$ tends to stabilize no-nodal gap. An yet another example is the equivalence between RPA [85] and fRG [86] results for heavily electron doped systems, in which only electron pockets remain - both approaches yield a d-wave gap with no nodes.

\section{DOPING DEPENDENCE OF THE COUPLINGS}

In this section I assume that SC is the leading instability and briefly review how the gap symmetry and structure evolve with doping. RG, fRG, and RPA results on the doping 
evolution of the gap agree with each other, and for definiteness I will use RPA approach combined with the LAHA (see Sec. III). The results differ for electron and hole dopings, and I consider them separately.

\section{A. Electron doping}

For small and moderate electron dopings, the FS consists of 4 pockets - two hole FS at $(0,0)$ and two electron FSs at $(0, \pi)$ and $(\pi, 0)$. Typical fits by LAHA, the parameters extracted from the fits, and the solutions in s-wave and d-wave channels are shown in Fig. 10 and in Table II. It turns out [66] that some system properties are sensitive to the choice of the parameters, but some are quite universal. The parameter-sensitive properties are the presence or absence of accidental nodes in the $s$-wave gap (although for most of parameters the gap does have nodes, as in Fig. 10) and the gap symmetry itself, because for most of input parameters and dopings $\lambda^{s}$ and $\lambda^{d}$ remain comparable as long as both hole and electron FSs are present (see Table II). That $d$-wave state is a strong competitor in 4-pocket systems has been first emphasized in Refs. [19,35]. The authors of [19] hinted that different FeSCs may have different symmetry even for the same topology of the FS.

The universal observation is that the driving force for attraction in both $s$-wave and $d$ wave channels is strong inter-pocket electron-hole interaction $\left(u_{h_{i} e}\right.$ and $\tilde{u}_{h_{i} e}$ terms) no matter how small the hole pockets are. The gap structure actually changes only little with doping as long as both hole and electron pockets are present.

TABLE II: Some of the LAHA parameters extracted from the LAHA fit in Figs. (10) and (11) for electron doping. Blocks (i) corresponds to Fig. (10), block (ii) corresponds to Fig. (11) (no hole pockets). From Ref [66].

\begin{tabular}{|c|c|c|c|c|c|c|c|c|c|}
\hline \multirow[b]{2}{*}{$s$-wave } & \multicolumn{6}{|c|}{ (i) } & \multicolumn{3}{|c|}{ (ii) } \\
\hline & $u_{h_{1} h_{1}}$ & $u_{h_{1} e}$ & $\alpha_{h_{1} e}$ & $u_{e e}$ & $\alpha_{e e}$ & $\lambda_{s}$ & $u_{e e}$ & $\alpha_{e e}$ & $\lambda_{s}$ \\
\hline \multirow{3}{*}{$d$-wave } & 0.8 & 0.79 & -0.19 & 0.91 & 0.05 & 0.25 & 3.65 & 0.20 & 0.1 \\
\hline & $\tilde{u}_{h_{1} h_{1}}$ & $\tilde{u}_{h_{1} e}$ & $\tilde{\alpha}_{h_{1} e}$ & $\tilde{u}_{e e}$ & $\tilde{\alpha}_{e e}$ & $\lambda_{d}$ & $\tilde{u}_{e e}$ & $\tilde{\alpha}_{e e}$ & $\lambda_{d}$ \\
\hline & 0.50 & -0.39 & -0.46 & -0.04 & 1.5 & 0.37 & -2.57 & 0.29 & 5.9 \\
\hline
\end{tabular}

The situation changes qualitatively once the hole pockets disappear (Fig. 11). It is clear 
from Table II that now the $d$-wave channel becomes the dominant one. Comparing the LAHA parameters for the two dopings, we see the reason: once the hole pockets disappear, a direct $d$-wave electron-electron interaction $\tilde{u}_{e e}$ becomes strong and attractive. The argument why this happens is as follows: [66] $\tilde{u}_{e e}$ is an antisymmetric combination of intra-pocket and interpocket electron-electron interactions $\tilde{u}_{e e}=u_{\text {intra }}^{e e}-u_{\text {inter }}^{e e}$. Both $u_{\text {inter }}^{e e}$ and $u_{\text {intra }}^{e e}$ are positive (repulsive), but the sign of $\tilde{u}_{e e}$ depends on the interplay between $u_{\text {inter }}^{e e}$ and $u_{\text {intra }}^{e e}$. As long as hole FSs are present, SF are peaked near $\mathbf{q}=(0, \pi)$ and $(\pi, 0)$, which are an equal distance from the relevant momenta $\mathbf{q}=0$ for $u_{\text {intra }}^{e e}$ and $\mathbf{q}=(\pi, \pi)$ for $u_{\text {inter. }}^{e e}$. In this situation, $u_{\text {intra }}^{e e}$ and $u_{\text {inter }}^{e e}$ remain close in magnitude, and $\tilde{u}_{e e}$ is small. Once the hole pockets disappear, the peak in the RPA spin susceptibility shifts towards $(\pi, \pi) \quad$ [85] and $u_{\text {inter }}^{e e}$ increases more due to the SF component than $u_{\text {intra }}^{e e}$. A negative $u_{\text {intra }}^{e e}-u_{\text {inter }}^{e e}$ then gives rise to a "plus-minus" gap on the two electron FSs. The gap changes sign under $k_{x} \rightarrow k_{y}$ and therefore has $d_{x^{2}-y^{2}}$ symmetry. This pairing mechanism is essentially identical to spin-fluctuation scenario for d-wave pairing in the cuprates [75].

A different proposal has been put forward in Refs. [82] and [83]. These authors argued that the gap symmetry may be nodeless $s$-wave (equal sign of the gap on the pockets at $(0, \pi)$ and $\pi, 0)$. According to $[82,83]$, s-wave pairing emerges, in some range of parameters, if one uses for electron-electron interaction the orbital version of the $J_{1}-J_{2}$ model. Yet another proposal for strongly electron-doped FeSCs is $s^{++}$pairing driven by orbital fluctuations [84].

TABLE III: Some of LAHA parameters extracted from the fits in Figs. 12 and 13 for hole doping. Block (i) corresponds to Fig. 12 (hole and electron pockets are present), block (ii) corresponds to Fig. 13 ( no electron pockets).

\begin{tabular}{|c|c|c|c|c|c|c|c|c|c|c|}
\hline \multirow[b]{2}{*}{$s-$ wave } & \multicolumn{5}{|c|}{ (i) } & \multicolumn{5}{|c|}{ (iii) } \\
\hline & $u_{h_{1} h_{1}}$ & $u_{h_{1} e}$ & $\alpha_{h_{1} e}$ & $u_{e e}$ & $\lambda_{s}$ & $u_{h_{1} h_{1}}$ & $u_{h_{1} h_{2}}$ & $u_{h_{1} h_{3}}$ & $u_{h_{3} h_{3}}$ & $\lambda_{s}$ \\
\hline \multirow{3}{*}{$d-w a v e$} & 0.0 .86 & 0.92 & -0.18 & 1.00 & 0.58 & 0.67 & 0.8 & 0.29 & 1.37 & 0.13 \\
\hline & $\tilde{u}_{h_{1} h_{1}}$ & $\tilde{u}_{h_{1} e}$ & $\tilde{\alpha}_{h_{1} e}$ & $\tilde{u}_{e e}$ & $\lambda_{d}$ & $\tilde{u}_{h_{1} h_{1}}$ & $\tilde{u}_{h_{1} h_{2}}$ & $\tilde{u}_{h_{1} h_{3}}$ & $\tilde{u}_{h_{3} h_{3}}$ & $\lambda_{d}$ \\
\hline & 0.51 & -0.45 & -0.48 & 0.07 & 0.31 & 0.36 & -0.5 & -0.02 & -0.17 & 0.11 \\
\hline
\end{tabular}




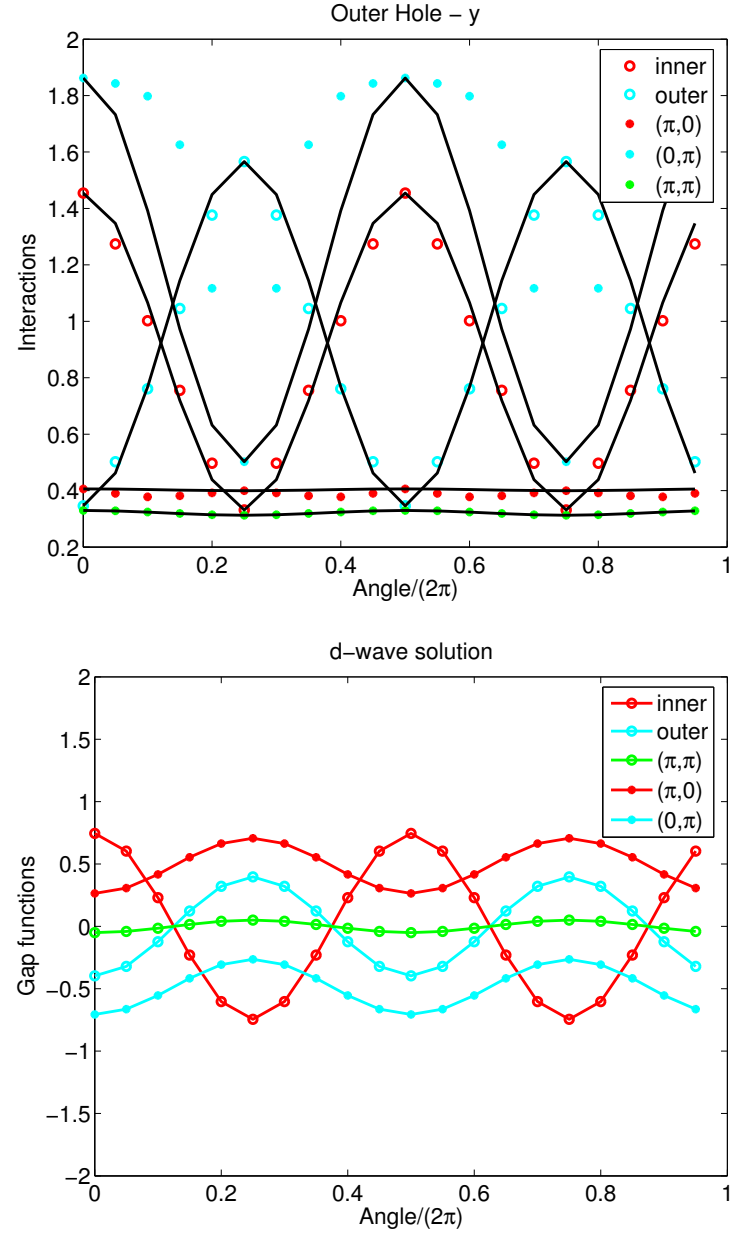

FIG. 12: Representative case of small/moderate hole doping, when both hole and electron pockets are present. Panel a - the FS, panel b - representative fits of the interactions by LAHA (the dots are RPA results, the lines are LAHA expressions, Eqs (9)-(13)). Panels c and d - the eigenfunctions in $s$-wave and $d$-wave channels for the largest $\lambda^{s}$ and $\lambda^{d}$. From Ref. [66].

\section{B. Hole doping}

For small and moderate hole doping, the FS contains 5 pockets - two hole pockets at $(0,0)$, two electron pockets at $(0, \pi)$ and $(\pi, 0)$, and one more hole pocket at $(\pi, \pi)$. Representative FSs for hole doping, typical fits by LAHA, the parameters extracted from the fit, and the solutions in s-wave and d-wave channels are shown in Fig. 12 and in Table III. Just like for electron doping, there are universal and parameter-sensitive features. The parametersensitive property is again the presence or absence of accidental nodes in the $s$-wave gap along the electron FSs, although for most of the parameters, the gap does not have nodes 

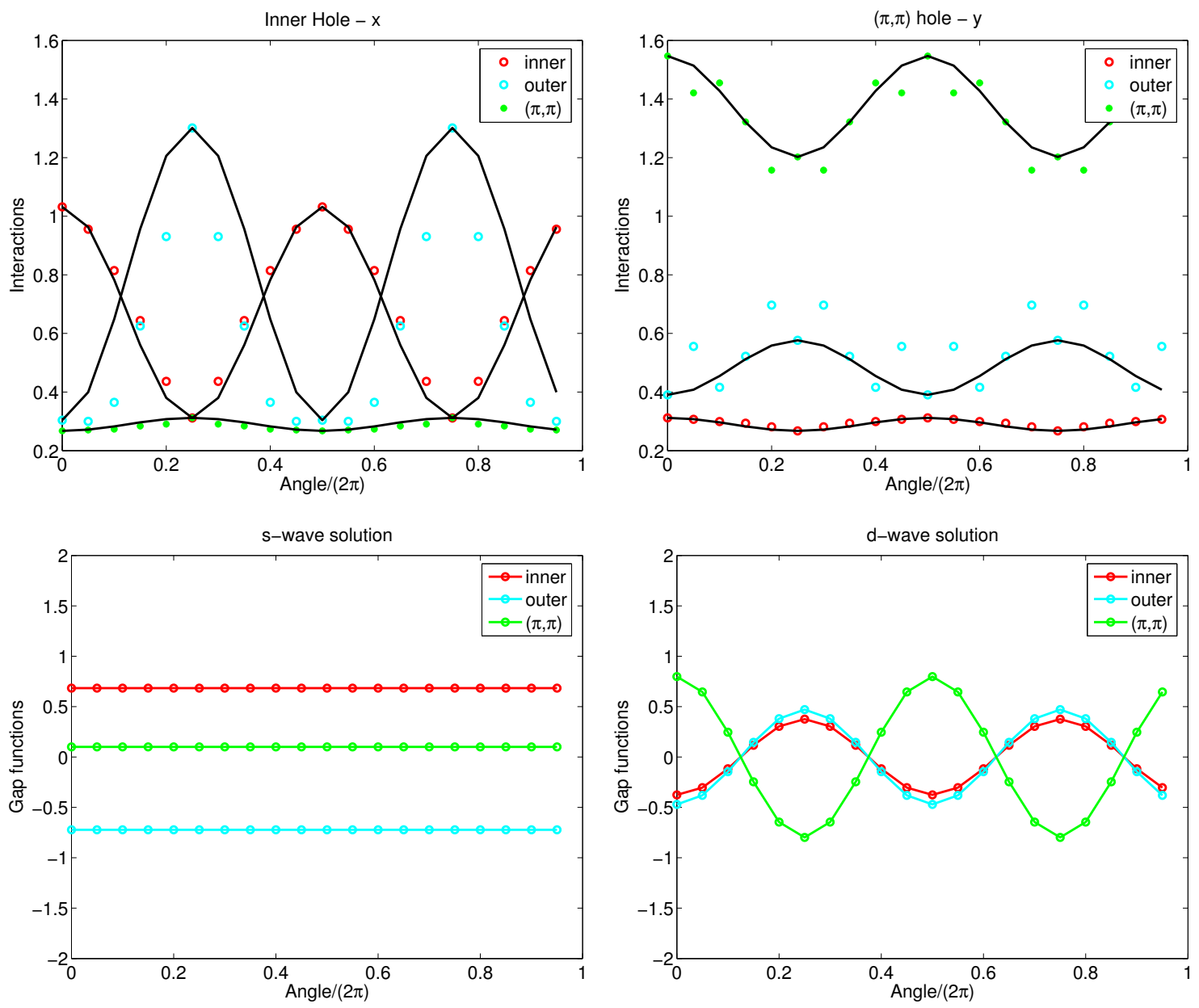

FIG. 13: The fits of the RPA interactions by LAHA and the structure of $s$-wave and $d$-wave gaps in for strong hole doping $(\mu=-0.30 \mathrm{eV})$, when only hole FSs are present. From Ref. [66].

(see Fig. 12) because the total $u_{h e}$ increases once it acquires an additional contribution $u_{h_{3} e}$.

There are two universal features. First, the $s$-wave eigenvalue is enhanced relative to a $d$-wave one and becomes the leading instability as long as both hole and electron pockets are present. Second, the driving force for the attraction in both $s$ - and $d$ - channels is again strong inter-pocket electron-hole interaction ( $u_{h e}$ and $\tilde{u}_{h e}$ terms), no matter how small electron pockets are.

The situation again changes rapidly once electron pockets disappear, see Fig. 13. Now electron-hole interaction becomes irrelevant, and the attractive pairing interaction may only be due to intra and inter-pocket interactions involving hole pockets. LAHA analysis shows [66] that, at least for in some range of parameters, there is an attraction in both $s$-wave and $d$-wave channels, and furthermore $\lambda_{d} \approx \lambda_{s}$, see Fig. 13 The near-equivalence of $s$-wave and 
$d$-wave eigenvalues was also found in recent unrestrictive RPA study [88]. Within LAHA, the attractive $\lambda_{s}$ is due to strong intra-pocket interaction between the two hole pockets centered at $(0,0)$. The $s$-wave gap then changes sign between these two hole pockets. The gap along $(\pi, \pi)$ pocket is induced by a weaker inter-pocket interaction and is much smaller. LAHA neglects $\cos 4 n \phi$ gap variations along the hole FSs (i.e., $s$-wave gaps are treated as angle-independent), but the theory can indeed be extended to include these terms.

The attractive $\lambda_{d}$ emerges by two reasons. First, the $d$-wave intra-pocket interaction $\tilde{u}_{h_{3} h_{3}}$ becomes negative, second, the inter-pocket interaction $\tilde{u}_{h_{1} h_{2}}$ between the two pockets at $(0,0)$ becomes larger in magnitude than repulsive $\tilde{u}_{h_{1} h_{1}}$ and $\tilde{u}_{h_{2} h_{2}}$ (see Table III). The solutions with $\lambda_{d}>0$ then exist separately for FSs $h_{1,2}$ and for $h_{3}$, the residual inter-pocket interaction just sets the relative magnitudes and phases between the gaps at $h_{3}$ and $h_{1,2}$. The $d$-wave gap with the same structure has been obtain in the fRG analysis at large hole doping [87]. That study found less competition with $s$-wave than in PRA-based studies.

\section{EXPERIMENTAL SITUATION}

Experimental study of FeSCs is one of the major research topics in condensed-matter physics since 2008 and several detailed reviews have already appeared in the literature [15, 16,18,24,25]. Here I briefly discuss the experimental situation concerning the symmetry and the structure of the SC gap.

As of today, there is no "smoking gun" experiment which would carry the same weight as phase-sensitive measurements of $d_{x^{2}-y^{2}}$ gap symmetry in the cuprates [33]. Still, there is enough experimental data to minimize the number of possible gap structures.

The theoretical proposals for the gap symmetry and structure are summarized in Sec. VIII. The proposed symmetry is different for weakly/moderately doped systems with hole and electron FSs and for strongly doped systems where FSs of only one type are present. It is then instructive to consider weak/moderate and strong doping separately.

\section{A. Moderate doping, gap symmetry}

The candidates are s-wave (either $s^{ \pm}$or $s^{++}$) or $d_{x^{2}-y^{2}}$ gap. The two behave very differently along the hole FSs centered at $(0,0)$ - s-wave gap is nodeless with $\cos 4 \phi$ vari- 
ations, while $\mathrm{d}$-wave gap has nodes along $k_{x}= \pm k_{y}$. ARPES measurements, both from synchrotron [55,89-91] and using laser light [42], show quite convincingly that the gap along hole FSs is nodeless in both hole and electron-doped FeSCs. This unambiguously selects an s-wave. Additional evidence in support of s-wave pairing comes from very flat low- $\mathrm{T}$ behavior of the penetration depth in the highest $T_{c} 1111$ FeSCs systems [92].

\section{B. Moderate doping, $s^{ \pm}$vs $s^{++}$}

The distinction between $s^{ \pm}$and $s^{++}$gaps is a more subtle issue, particularly given that both belong to the same $A_{1 g}$ representation and also because in general $A_{1 g}$ gap on electron pockets may have strong oscillating component. In general, the gaps on electron and hole

FSs have non-equal magnitudes, and the issue whether the gap is $s^{ \pm}$or $s^{++}$reduces to whether the gap averaged over an electron FS has the same sign or opposite sign than the gap averaged over a hole FS. This is not a fundamental symmetry issue and, moreover, when $\cos 2 \phi$ oscillations are strong, one may switch from equal to opposite signs of the averaged gaps by a small change of parameters [64] or by adding impurities. [93] Still, when oscillations are not very strong, whether the eigenfunction has $s^{ \pm}$or $s^{++}$character is essential because it determines, to a large extent, whether the pairing is driven by spin or by orbital fluctuations (see Sec.IV).

The experimental data most frequently cited in support of $s^{ \pm}$gap is the observation of a magnetic resonance in neutron scattering [13,94]. If, as many researchers believe, the resonance is a spin exciton, it exists at a momentum $Q$ if the gaps at FS momenta $k_{F}$ and $k_{F}+Q$ are of opposite sign. Experimentally, the resonance is observed [13,94] near $Q=(\pi, \pi)$ in the folded BZ, which in this zone is precisely the distance between electron and hole FSs. The excitonic resonance then exists if the gap changes sign between hole and electron pockets and does not exist if the gap doesn't change sign. A similar reasoning has been used in identifying the the resonance seen in the cuprates with a fingerprint of $d_{x^{2}-y^{2}}$ gap symmetry [95]

From experimental perspective, the neutron peak is the resonance if it is narrow and is located below twice the gap value. The argument made by the supporters of $s^{++}$scenario [36] is that the observed neutron peak is more broad than the resonance seen in the cuprates, and that there is no firm evidence that the peak energy is below $2 \Delta$ for the minimum gap. 
For $s^{++}$gap structure, there is no resonance, but there is a redistribution of the neutron spectral weight immediately above $2 \Delta$ what gives rise to a local maximum in the magnetic structure factor $[36,96,97]$. Still, the majority of researchers do believe that the observed neutron peak is a resonance, and the fact that it is quite broad is at least partly due to $\cos 2 \phi$ gap variations along the electron FSs [97].

Another rather strong evidence in support of $s^{ \pm}$gap is the observed variation of the quasiparticle interference pattern in a magnetic field [98] although the interpretation of the data has been subject of debates [99]. It was also argued [100] that the very presence of the co-existence region between $\mathrm{SC}$ and stripe magnetism in FeSCs is a fingerprint of an $s^{ \pm}$ gap, because for $s^{++}$gap a first order transition between a pure magnetic and a pure SC state is a much more likely scenario.

\section{Moderate doping, nodal vs no-nodal $s^{ \pm}$gap}

Assume for definiteness that the pairing is driven by spin fluctuations and the gap has $s^{ \pm}$ structure. In 2D scenario, such gap has $\cos 2 \phi$ variations along electron FSs, which, according to theory, can be rather strong, particularly in electron-doped FeSCs. Experimental data show that, whether or not the gap is nodeless or has nodes, depends on the material, on the doping, and on whether SC co-exists with SDW order.

\section{Hole doping}

For hole-doped FeSCs (e.g. for $\mathrm{Ba}_{1-x} \mathrm{~K}_{x} \mathrm{Fe}_{2} \mathrm{As}_{2}$ ) the data indicate that the gap is nodeless, away from the co-existence region. This is consistent with the theory (see Sec. V). ARPES experiments do not show any angular variation of the gap along both hole and electron FSs $[55,89]$, but it is not entirely clear whether ARPES can at present distinguish between the gaps on the two electron FSs which in folded zone are both centered at $(\pi, \pi)$. Thermal conductivity data show that $\kappa / T$ tends to zero in the limit of $T=0$, in line with what one should expect for a nodeless SC [101]. Specific heat data also show non-nodal behavior [102]. The interpretation of the penetration depth data requires more care as the data do show a power-law behavior $\lambda(T)-\lambda(0) \propto T^{a}$ with $a \sim 2$ (Refs. [103]). Such a behavior is expected for a SC with point nodes, but it is also expected in a wide range of $T$ for a nodeless $s^{ \pm} \mathrm{SC}$ in 
the presence of modest inter-band scattering by non-magnetic impurities [104]. Penetration depth measurements on artificially irradiated samples [105] support the idea that the gap is nodeless and power-law $T^{a}$ behavior of $\lambda(T)-\lambda(0)$ is due to impurities.

\section{Electron doping}

For electron-doped FeSCs, e.g., 122 materials like $\mathrm{Ba}\left(\mathrm{Fe}_{1-x} \mathrm{Co}_{x}\right)_{2} \mathrm{As}_{2}$ or 1111 materials like $\mathrm{NdFeAsO}_{1-x} \mathrm{~F}_{x}$, ARPES shows no-nodal gap along hole FS [90,91], but there are no data on the gap along each of the two electron FSs. At optimal doping, the data on both thermal conductivity $[106,109]$ and penetration depth $[109,110]$ are consistent with no-nodal gap However, the data for overdoped $\mathrm{Ba}\left(\mathrm{Fe}_{1-x} \mathrm{Co}_{x}\right)_{2} \mathrm{As}_{2}$ indicate that gap nodes may develop: the behavior of $\lambda(T)$ becomes more steep, and $\kappa / T$ now tends to a finite value [106], expected for a $\mathrm{SC}$ with line nodes. The data also show $\sqrt{H}$ behavior of $\kappa$ in a magnetic field [106] also expected for a SC with line nodes [107], but it was argued that the behavior resembling $\sqrt{H}$ can be obtained even if $s^{ \pm}$gap has no nodes [108]. There is also clear anisotropy between in-plane conductivity and conductivity along $z$ direction, what was interpreted [106] as an indication that the nodes may be located near particular $k_{z}$. Specific heat data in overdoped $\mathrm{Ba}\left(\mathrm{Fe}_{0.9} \mathrm{Co}_{0.1}\right)_{2} \mathrm{As}_{2}$ were also interpreted as evidence for the nodes. [111]

The development of the nodes in $s^{ \pm}$gap upon electron doping is in line with the theory. The farther the system moves away from the SDW phase, the weaker is the increase of intraband electron-hole interaction and hence the stronger is the competition from intra-band repulsion. As I discussed in Sec.III), the gap adjusts to this change by increasing its $\cos 2 \phi$ component in order to effectively reduce the effect of the intra-band repulsion in the gap equation.

There is also experimental evidence for $\cos 2 \phi$ gap oscillations from the observed oscillations [112] of the field-induced component of the specific heat $C(H, T)$ in superconducting $\mathrm{FeTe}_{1-x} \mathrm{Se}_{x}(x \sim 0.5)$. The measured $C(H, T)$ oscillates with the direction of the applied field as $\cos 4 \phi$. In theory, such an oscillation is related to the behavior of $\Delta^{2}(\phi)$ (Ref. [62]), hence $\cos 2 \phi$ gap oscillations in $\Delta$ lead to $\cos 4 \phi$ oscillations in $C(H, T)$. The observed field and temperature dependence of the prefactor for $\cos 4 \phi$ term are consistent with the idea that the oscillations are caused by $\cos 2 \phi$ term in $\Delta$. These data were also interpreted as 
evidence for no-nodal gap because if $\cos 2 \phi$ gap oscillations were strong and the gap had nodes at accidental points, the behavior of $\Delta^{2}$ would be more complex than the observed $a+b \cos 4 \phi$.

For LiFeAs, which is undoped but has FS structure similar to electron-doped FeSCs, no-nodal behavior has been observed in ARPES [113], specific heat [114], penetration depth [115] and NMR [43,116] measurements. The NMR data, however, were interpreted [43] as evidence for a possible B-type $p$-wave gap $\left(p_{x}+i p_{y}\right)$. Whether this is the case remains an open issue. In any event, the evidence for a no-nodal gap in this material is overwhelming.

\section{Co-existence region}

Taken at a face value, thermal conductivity and penetration depth data indicate that the gap becomes nodal in the co-existence regime in both hole-doped and in electron-doped FeSCs. The most striking evidence comes from thermal conductivity $[101,106]$ - in the co-

existence regime $\kappa / T$ tends to a finite value at $T \rightarrow 0$ and shows $\sqrt{H}$ behavior, both typical for a SC with line nodes. There is no theoretical understanding at present why the SC gap develops nodes in the co-existence regime (for a toy two-pocket model theory predicts that the gap should have no-nodes [117]), so this is another open issue.

\section{Isovalent doping}

Electron or hole doping is not the only way to change the properties of FeSCs. Another route is to replace one pnictide with the other. The most common replacement is As $\rightarrow \mathrm{P}$. P-containing materials include the very first FeSC - LaFeOP, with $T_{c} \leq 5 K$ (Ref. [118]), the family $\mathrm{BaFe}_{2} \mathrm{As}_{1-x} \mathrm{P}_{x}$ with the highest $T_{c}$ around 30K (Ref. [119]), and LiFeP [120]. Penetration depth, thermal conductivity, specific heat, and NMR data [121] in these materials all show the behavior consistent with line nodes. In particular, $\kappa$ scales linearly with $T$ at low $T$ and displays $\sqrt{H}$ behavior in a magnetic field, and $\lambda(T)-\lambda(0)$ is also linear in $T$ down to very low $T$. Laser ARPES data show [42] that the gap along FS is nodeless, so the nodes likely are located on electron FSs.

On general grounds, the existence of the nodes on electron FSs is in line with theory 
predictions particularly as $\mathrm{BaFe}_{2} \mathrm{As}_{1-x} \mathrm{P}_{x}$ has the same structure of 4 cylindrical FSs as electron-doped FeSCs for which nodes are most likely. It has been argued [35] that a replacement of As by $\mathrm{P}$ changes the hight of a pnictide with respect to Fe plane, what effectively reduces inter-pocket electron-hole interaction, in which case the gap develops nodes to reduce the effect of intra-pocket repulsion. However, this argument is only suggestive, and it is not entirely clear at the moment why all P-based FeSCs have nodes. One way to analyze this semi-quantitatively is to study the correlation between $2 \Delta / T_{c}$ on the hole FS and the presence of the nodes on electron FSs. This study shows [122] that from this perspective P-based FeSCs are indeed the "best case" for the gap nodes.

Another open issue is the location of the nodes along z- direction. Oscillations of thermal conductivity with the direction of a magnetic field have been measured recently [123], and $\cos 4 \phi$ component of these oscillations has been interpreted using the modified $2 \mathrm{D}$ form $\Delta_{e}\left(k_{z}\right)=\Delta_{0}\left(1+\alpha\left(k_{z}\right) \cos 2 \phi\right)$. The best fit to the data yields $\alpha\left(k_{z}\right)>1$ for some $k_{z}$ and $\alpha\left(k_{z}\right)<1$ for others, in which case the nodes form patches along $k_{z}$. However, whether this is the only explanation of the data is debatable.

I caution that although the nodes on electron FSs are most likely, they have not been directly observed yet, so it is still possible that the nodes are located on a hole FS, near particular $k_{z}$, as some of 3D theories suggest [59]. Another possibility, which is also not entirely ruled out, is that the system behavior near the surface, probed by ARPES, is not the same as in the bulk. The probability that this is the case is not high, though, because ARPES data are obtained using a laser light which probes states located farther from the surface than in conventional synchrotron-based ARPES.

\section{Strongly doped FeSCs}

\section{Electron doping}

Strongly electron doped materials is recently discovered family of $\mathrm{A}_{x} \mathrm{Fe}_{2-y} \mathrm{Se}_{2}(A=$ $K, R b, C s) \quad[11,12]$ of which $\mathrm{K}_{0} .8 \mathrm{Fe}_{1.7} \mathrm{Se}_{2}$ is the most studied material. $T_{c}$ in $\mathrm{A}_{x} \mathrm{Fe}_{2-y} \mathrm{Se}_{2}$ is rather high, almost 40K. ARPES shows [12] that only electron FSs are present in $\mathrm{A}_{x} \mathrm{Fe}_{2-y} \mathrm{Se}_{2}$, while hole pockets are at least $60 \mathrm{meV}$ from the FS, although hole dispersion above $60 \mathrm{meV}$ is still clearly visible in ARPES. Two electron FSs are at $(0, \pi)$ and $(\pi, 0)$, 
like in other FeSCs, and there is, possibly, another electron FS at $(0,0)$. RPA, LAHA and fRG calculations for these systems predict that the gap should have a d-wave symmetry, at least for the case when the FSs are only at $(0, \pi)$ and $(\pi, 0)$. A d-wave symmetry in this situation means that the gaps on the two electron FSs behave as $\Delta_{0}( \pm 1+\alpha \cos 2 \phi)$, and all calculations yield $\alpha<1$, i.e., no nodes (neglecting 3D effects). The theoretical alternative is $s^{++}$symmetry by one reason [84] or the other $[82,83]$. At present, both ARPES and specific heat data point that the gap is nodeless, at least for most of $k_{z}$ values, but whether the gap is an $s$-wave or a nodeless $d$-wave remains to be seen.

\section{Hole doping}

Strongly hole doped system is $\mathrm{KFe}_{2} \mathrm{As}_{2}\left(T_{c}=3 K\right)$ which is at the opposite end from parent $\mathrm{BaFe}_{2} \mathrm{As}_{2}$ in the family of $\mathrm{K}_{x} \mathrm{Ba}_{1-x} \mathrm{Fe}_{2} \mathrm{As}_{2}$. According to ARPES [40], this system has no electron pockets and has hole pockets at $(0,0)$. There may also be additional hole pockets, but this is not entirely clear. Both thermal conductivity and penetration depth measurements clearly point to nodal behavior [41]. There is, however, no "smoking gun" symmetry-sensitive measurement, so whether the gap is a d-wave or an $s$-wave with nodes due to strong $\cos 4 \phi$ gap component on one of the FSs remains an open issue

\section{E. Summary}

Overall, the agreement between theory and experiment with respect to gap symmetry and structure is reasonably good. Theory predicts that the gap symmetry in weakly and moderately doped FeSCs is an s-wave. This is consistent with ARPES data. Many theorist argue that an s-wave gap changes sign between hole and electron FSs. Quasiparticle interference and neutron scattering data are consistent with this picture, if, indeed, the neutron peak is

the resonance. Further, theory predicts that $s^{ \pm}$gap has oscillations along electron FS, and these oscillations give rise to accidental nodes, which are more likely for systems with 2 hole and 2 electron cylindrical FSs than in systems with an additional hole cylindrical FS. This is also generally consistent with the experiments, although at this moment the agreement is more qualitative than quantitative. For strongly electron-doped FeSCs, the theory based on RPA, LAHA, and fRG predicts a nodeless d-wave gap, except, perhaps, the region near 
$k_{z}=\pi / 2$. This is neither confirmed not disproved by the experiments. For strongly holedoped FeSCs, with only hole pockets, experiments point to the presence of the nodes, but whether the gap is a $d$-wave or a nodal $s$-wave has not been firmly established. Finally, in the co-existence region of SC + SDW, theory prediction for a 2-band toy model is a nodeless $s^{ \pm}$gap. This is consistent with the data for weak SDW order, but apparently inconsistent with the data for FeSCs with strong SDW order, which show behavior consistent with the nodes in the SC gap. What is the gap structure deep in the co-existence region remains to be seen.

\section{CONCLUSION}

The analysis of the gap symmetry and structure in FeSCs is a fascinating subject because of multi-orbital/multi-band nature of these materials. It is now well understood that in multi-band systems a conventional notion that s-wave gap is nodeless, d-wave has 4 nodes, etc, does not work, and s-wave gap may have nodes, while d-wave gap may remain nodeless. Furthermore, nodes may appear or disappear, depending on doping and other external conditions. Another peculiarity of FeSCs is a close proximity between pairing eigenvalues $\lambda_{i}$ from different symmetry representations, e.g., $A_{1 g}$ and $B_{1 g}$. Because of proximity, even the gap symmetry may change upon doping or other external perturbation. Also, below $T_{c}$, the system may lower the energy by moving into a mixed state with, e.g., $s+i d$ gap function. To analyze this issue, one needs to go beyond the analysis presented in this review and solve the full non-linear gap equation.

The existence of superconductivity at the "end points" of the phase diagram, when only hole pockets or only electron pockets are present, is another fascinating issue. There is reasonably good understanding of a d-wave pairing at strong hole doping, but there are competing proposals for the gap symmetry at strong electron doping, and so far experiments cannot distinguish between these proposals. Finally, the gap structure in the co-existence regime with SDW is yet another fascinating unexplored issue, and comprehensive analysis of $\mathrm{SC}$ in the co-existence phase is clearly called for. 


\section{Acknowledgements}

I acknowledge helpful discussions with L. Benfatto, A. Bernevig, S. Budko, P. Canfield, A. Carrington, A. Coldea, L. Digiorgi, R. Fernandes, S. Graser, H. Ding, W. Hanke, P. Hirschfeld, K. Honerkamp, D. Efremov, I. Eremin, J. Knolle, H. Kontani, K. Kuroki, DH. Lee, T. Maier, S. Maiti, Y. Matsuda, I. Mazin, K. Moller, R. Prozorov, D. Scalapino, T. Shibauchi, Q. Si, J. Schmalian, H. Takagi, Z. Tesanovic, R. Thomale, M. Vavilov, A. Vorontsov, and H.H. Wen. I am thankful to S. Sachdev for careful reading of the manuscript and useful comments. This work was supported by NSF-DMR-0906953.

\section{SUMMARY POINTS}

- For weakly and moderately electron-doped FeSCs, $s^{ \pm}$and $d_{x^{2}-y^{2}}$ pairing channels are nearly degenerate. The driving force for the pairing in both channels is inter-pocket electron-hole interaction, enhanced by spin fluctuations. d-wave gap has nodes at $k_{x}= \pm k_{y}$ on the hole FSs, $s^{ \pm}$gap has $\cos 2 \phi$ variations on electron FSs and may have nodes. The probability that $s^{ \pm}$gap has nodes increases with electron doping.

- For strongly electron doped FeSCs, when only electron pockets remain, some results show that the gap has a $d$-wave symmetry, which in this situation implies that angleindependent component of the gap changes sign between the two electron pockets. The driving mechanism for the pairing is a direct $d$-wave attraction between electron pockets, again enhanced by spin fluctuations. The gap is nodeless, but may acquire nodes near $k_{z}=\pi / 2$ when $3 \mathrm{D}$ effects and/or hybridization between electron pockets are included. A competing theory proposal, based on orbital $J-1-J-2$ model, is that the gap is sign-preserving s-wave.

- For weakly and moderately hole-doped FeSCs, $s^{ \pm}$pairing is the dominantinstability. The driving force is again inter-pocket electron-hole interaction, enhanced by spin fluctuations. The gap has $\cos 2 \phi$ variation along electron FSs but likely does not have nodes.

- For strongly hole-doped FeSCs, when only hole FSs remain, the gap symmetry may be $d$-wave, with nodes at $k_{x}= \pm k_{y}$ on all hole FSs. The d-wave pairing mechanism is 
the combination of a d-wave attraction within the hole pocket at $(\pi, \pi)$ and a strong intra-pocket d-wave interaction between the two hole pockets centered at $(0,0)$. A competing theory proposal is that the gap is s-wave, with potential nodes due to strong $\cos 4 \phi$ variation along hole FSs centered at $(0,0)$.

- For real 3D systems, one scenario is that the 2D picture survives, and nodes, if present, are "vertical" nodes, located on electronic FSs at given $k_{x}$ and $k_{y}$ but arbitrary $k_{z}$. Another scenario is that nodes on electron FSs exists only in some ranges of $k_{z}$. And the third, more radical scenario, is that the nodes appear as "horizontal" nodes near particular $k_{z}$, either on electron or on hole FSs.

- An alternative scenario for all FeSCs is that the gap is a conventional, sign-preserving $s^{++}$gap, but still may have $\cos 2 \phi$ oscillations on electron FSs. Such a gap appears if inter-pocket electron-hole interaction $u_{h e}$ is negative (attractive) and is enhanced by charge (orbital) fluctuations and by phonons

\section{A LIST OF FUTURE ISSUES}

- What would be the "smoking gun" experiment to distinguish between $s^{ \pm}$and $s^{++}$ gap?

- Why all P-based FeSCs appear to have nodes, and where these nodes are located?

- What is the structure of the SC gap in the co-existence region with SDW order?

- What is the pairing mechanism and the gap symmetry and structure in heavily electron-doped $\mathrm{K}_{x} \mathrm{Fe}_{2-y} \mathrm{Se}_{2}$ and in heavily hole-doped $\mathrm{KFe}_{2} \mathrm{As}_{2}$ ?

- Is there a mixed $s+i d$ SC state in FeSCs at $T=0$ ?

- Is there a FeSC with a p-wave symmetry?

1 Y. Kamihara, T. Watanabe, M. Hirano, H. Hosono, J. Am. Chem. Soc. 130, 3296(2008).

2 X. H. Chen, T. Wu, G. Wu, R. H. Liu, H. Chen, D. F. Fang, Nature 453, 761(2008). 
3 G. F. Chen, Z. Li, D. Wu, G. Li, W. Z. Hu et al Phys. Rev. Lett. 100, 247002 (2008).

4 Z.-A. Ren, G.-C. Che, X.-L. Dong, J. Yang, W. Lu et al Europhys. Lett. 83, 17002(2008)

5 M. Rotter, M. Tegel, D. Johrendt, Phys. Rev. Lett. 101, 107006 (2008)

6 K. Sasmal, B. Lv, B. Lorenz, A. M. Guloy, F. Chen, Y.-Y. Xue, and C.-W. Chu, Phys. Rev. Lett. 101, 107007 (2008),

7 N. Ni, A. Thaler, J. Q. Yan, A. Kracher, E. Colombier, S. L. Bud'ko, P. C. Canfield, Phys. Rev. B 82, 024519 (2010).

8 Y. Nakai, T. Iye, S. Kitagawa, K. Ishida, H. Ikeda, S. Kasahara, H. Shishido, T. Shibauchi, Y. Matsuda, T. Terashima, Phys. Rev. Lett. 105, 107003 (2010).

9 X.C.Wang, Q.Q. Liu, Y.X. Lv, W.B. Gao, L.X.Yang, R.C.Yu, F.Y.Li, and C.Q. Jin, arXiv:0806.4688v3; S. V. Borisenko, V. B. Zabolotnyy, D. V. Evtushinsky, T. K. Kim, I. V. Morozov et al Phys. Rev. Lett. 105, 067002 (2010).

10 Y. Mizuguchi, F. Tomioka, S. Tsuda, T. Yamaguchi, and Y. Takano, Appl. Phys. Lett. 93, 152505 (2008), F. C. Hsu et al., Proc. Natl. Acad. Sci. U.S.A. 105, 14262 (2008), M. H. Fang, H. M. Pham, B. Qian, T. J. Liu, E. K. Vehstedt, Y. Liu, L. Spinu, and Z. Q. Mao, Phys. Rev. B 78, 224503 (2008), G. F. Chen, Z. G. Chen, J. Dong, W. Z. Hu, G. Li, X. D. Zhang, P. Zheng, J. L. Luo, and N. L. Wang, Phys. Rev. B 79, 140509(R) (2009).

11 J. Guo, S. Jin, G. Wang, S. Wang, K. Zhu, T. Zhou, M. He, and X. Chen, Phys. Rev. B 82, $180520(\mathrm{R})(2010)$.

12 T. Qian, X.-P. Wang, W.-C. Jin, P. Zhang, P. Richard, G. Xu, X. Dai, Z. Fang, J.-G. Guo, X.-L. Chen, H. Ding, Phys. Rev. Lett. 106, 187001 (2011).

13 For the latest results on magnetic measaurements, see D. S. Inosov, J. T. Park, P. Bourges, D. L. Sun, Y. Sidis, A. Schneidewind, K. Hradil, D. Haug, C. T. Lin, B. Keimer, and V. Hinkov, Nature Physics 6, 178-181 (2010) and references therein.

14 Y. Kamihara, T. Watanabe, M. Hirano, and H. Hosono, J. Am. Chem. Soc. 128, 10012 (2006).

15 D.C. Johnston, Adv. Phys., 59, 803 (2010)

16 J-P Paglione and R.L. Greene, Nature Phys. 6, 645 (2010).

17 I.I. Mazin, Nature 464, 183 (2010).

18 H.H. Wen and S. Li, Annu. Rev. Condens. Matter Phys., 2, 121 (2011).

19 S. Graser, T. A. Maier, P. J. Hirshfeld, D. J. Scalapino, New J. Phys. 11, 025016 (2009).

20 A. F. Kemper, T. A. Maier, S. Graser, H.-P. Cheng, P. J. Hirschfeld, and D. J. Scalapino, New 
J. Phys. 12, 073030 (2010).

21 K. Kuroki, H. Usui, S. Onari, R. Arita, and H. Aoki, Phys. Rev. B 79, 224511 (2009).

22 A. V. Chubukov Physica C 469, 640(2009).

23 I.I. Mazin and J. Schmalian, Physica C, 469, 614 (2009).

24 D.N. Basov and A.V. Chubukov, Nature Physics 7, 241 (2011).

25 P.J. Hirschfeld, M.M. Korshunov, and I.I. Mazin, arXiv:1106.3712.

26 H. Kamerlingh Onnes. Comm. Phys. Lab. Univ. Leiden (1911).

27 J. Bardeen, L. N. Cooper, and J. R. Schrieffer. Phys. Rev., 108, 1175 (1957).

28 J. Kortus, I. I. Mazin, K. D. Belashchenko, V. P. Antropov, and L. L. Boyer. Phys. Rev. Lett., 86, 4656 (2001).

29 P. W. Anderson and P. Morel. Phys. Rev., 123, 1911-1934 (1961).

30 J. G. Bednorz and K. A. Müller, Zeitschrift für Physik B Condensed Matter, 64, 189 (1986).

31 E. M. Lifshitz and L. P. Pitaevski, Statistical Physics, (Pergamon Press, 1980).

32 W. Kohn and J. M. Luttinger, Phys. Rev. Letters, 15, 524 (1965).

33 see e.g., B. Goss Levi, Physics Today, January 1996, Page 19 and references therein.

34 I. I. Mazin, D. J. Singh, M. D. Johannes, and M. H. Du, Phys. Rev. Lett. 101, 057003 (2008).

35 K. Kuroki, S. Onari, R. Arita, H. Usui, Y. Tanaka, H. Kontani, and H. Aoki Phys. Rev. Lett. 101, 087004 (2008).

36 Seiichiro Onari and Hiroshi Kontani, Phys. Rev. Lett. 103, 177001 (2009).

37 W.G. Yin, C. C. Lee, and Wei Ku, Phys. Rev. Lett. 105, 107004 (2010).

38 R. Fernandes, A.V. Chubukov, I. Eremin, J. Knolle, and J. Schmalian, in preparation.

39 P. A. Lee and X.-G. Wen, Phys. Rev. B 78, 144517 (2008).

40 T. Sato, K. Nakayama, Y. Sekiba, P. Richard, Y.-M. Xu et al Phys. Rev. Lett. 103, 047002 (2009); T. Terashima et al., J. Phys. Soc. Japan 79, 053702 (2010).

41 J. K. Dong, S. Y. Zhou, T. Y. Guan, H. Zhang, Y. F. Dai et al Phys. Rev. Lett. 104, 087005 (2010); K. Hashimoto, A. Serafin, S. Tonegawa, R. Katsumata, R. Okazaki et al., Phys. Rev. B 82, 014526 (2010).

42 T. Shimojima, F. Sakaguchi, K. Ishizaka, Y. Ishida, T. Kiss et. al., (unpublished)

43 P. M. R. Brydon, M. Daghofer, C. Timm, J. van den Brink, Phys. Rev. B 83, 060501 (2011).

44 H. Ding, P. Richard, K. Nakayama, K. Sugawara, T. Arakane et al Europhys. Lett., 83, 47001 (2008). 
45 T. Y. Chen, Z. Tesanovic, R. H. Liu, X. H. Chen, and C. L. Chien, Nature, 453, 1224 (2008).

46 A. D. Christianson, E. A. Goremychkin, R. Osborn, S. Rosenkranz, M. D. Lumsden, C. D. Malliakas, I. S. Todorov, H. Claus, D. Y. Chung, M. G. Kanatzidis, R. I. Bewley, and T. Guidi, Nature, 456, 930 (2008).

47 L. Malone, J. D. Fletcher, A. Serafin, A. Carrington, N. D. Zhigadlo, Z. Bukowski, S. Katrych, and J. Karpinski, Phys. Rev. B 79, 140501(R) (2009).

48 S. Kasahara et al, Phys. Rev. B 81, 184519, (2010).

49 J. D. Fletcher, A. Serafin, L. Malone, J. Analytis, J.-H. Chu, A. S. Erickson, I. R. Fisher, and A. Carrington, Phys. Rev. Lett. 102, 147001 (2009).

50 R. T. Gordon, N. Ni, C. Martin, M. A. Tanatar, M. D. Vannette, H. Kim et al Phys. Rev. Lett., 102, 127004 (2009).

51 C. Martin, R. T. Gordon, M. A. Tanatar, H. Kim, N. Ni, S. L. Bud'ko et al Phys. Rev. B 80, 020501(R) (2009).

52 L. Luan, O. M. Auslaender, T. M. Lippman, C. W. Hicks, B. Kalisky et al Phys. Rev. B 81, 100501(R) (2010).

53 M. Yi, D. H. Lu, J. G. Analytis, J.-H. Chu, S.-K. Mo et al Phys. Rev. B 80, 024515 (2009)

54 S. E. Sebastian, J. Gillett, N. Harrison, P. H. C. Lau, C. H. Mielke, and G. G. Lonzarich, J. Phys.: Condens. Matter 20 422203(2008).

55 D. V. Evtushinsky, D. S. Inosov, V. B. Zabolotnyy, M. S. Viazovska, R. Khasanov et al New J. Phys. 11, 055069 (2009);

56 D.J. Singh and M.-H. Du, Phys. Rev. Lett. 100, 237003 (2008); M.J. Calderon, B. Valenzuela, and E. Bascones, Phys. Rev. B 80, 094531 (2009).

57 L. Boeri, O. V. Dolgov, and A. A. Golubov, Phys. Rev. Lett. 101, 026403 (2008)

58 I.I. Mazin, arXiv:1102.3655 (unpublished).

59 S. Graser, A. F. Kemper, T. A. Maier, H.-P. Cheng, P. J. Hirschfeld, and D. J. Scalapino, Phys. Rev. B 81, 214503 (2010).

60 M. Norman, Physics 1, 21 (2008).

61 see e.g. R. M. Fernandes, L. H. VanBebber, S. Bhattacharya, P. Chandra, V. Keppens et al Phys. Rev. Lett. 105, 157003 (2010); C. Xu, M. Mueller, and S. Sachdev, Phys. Rev. B 78, 020501(R) (2008); C. Fang, H. Yao, W.-F. Tsai, J.P. Hu, and S. A. Kivelson, Phys. Rev. B 77 224509 (2008). 
62 A.B.Vorontsov and I.Vekhter, Phys. Rev. Lett. 105, 187004 (2010); A.V. Chubukov and I. Eremin, Phys. Rev. B 82, 060504(R) (2010).

63 Ar. Abanov, A. V. Chubukov, and M. R. Norman, Phys. Rev. B 78, 220507(R) (2008).

64 S. Maiti and A.V. Chubukov, Phys. Rev. B 82, 214515 (2010).

65 Fa Wang, H. Zhai, Y. Ran, A. Vishwanath, and D-H Lee, Phys. Rev. Lett. 102, 047005 (2009); C. Platt, C. Honerkamp, and Werner Hanke, New J. Phys. 11, 055058 (2009); R. Thomale, C. Platt, J-P. Hu, C. Honerkamp, and B. A. Bernevig, Phys. Rev. B 80, 180505 (2009). For general discussion on fRG see, e.g., M. Salmhofer et al, Prog. Theor. .Phys. 112, 943 (2004).

66 S. Maiti, M.M. Korshunov, T.A. Maier, P.J. Hirschfeld, and A.V. Chubukov, arXiv:1104.1814.

67 A. V. Chubukov, M. G. Vavilov, A. B. Vorontsov, Phys. Rev. B 80, 140515(R)(2009).

68 Q. Si and E. Abrahams, Phys. Rev. Lett. 101, 076401 (2008).

69 C. Cao, P.J. Hirschfeld, H.-P. Cheng, Phys. Rev. B 77, 220506(R) (2008).

70 A. V. Chubukov, D. Efremov, and I. Eremin, Phys. Rev. B 78, 134512 (2008).

71 W. L. McMillan, Phys. Rev. 167, 331 (1968); N.N. Bogolubov, V.V. Tolmachev, and D.V. Shirkov, Consultants Bureau, 1959.

72 Daniel Podolsky, Hae-Young Kee, and Yong Baek Kim, Europhysics Letters 88, 17004 (2009).

73 K. Le Hur and T. M. Rice, Annals of Physics 324 (2009) 1452

74 N.F. Berk and J. R. Schrieffer, Phys. REv. Lett 17, 433 (1966).

75 See, e.g., P. Monthoux and D. Pines, Phys. Rev. B 47, 6069 (1993); D.J. Scalapino, Phys. Rep. 250, 329 (1995); D. Manske, I. Eremin, and K. H. Bennemann, Phys. Rev. B 62, 13922 (2000); A. Abanov, A. V. Chubukov, and J. Schmalian, Adv. Phys. 52, 119 (2003).

76 D.L. Maslov and A.V. Chubukov, Phys. Rev. B 81, 045110 (2010).

77 L. Benfatto, M. Capone, S. Caprara, C. Castellani, and C. Di Castro, Phys. Rev. B 78, 140502(R) (2008).

78 J-H. Chu, J G. Analytis, K. De Greve, P. L. McMahon, Z. Islam, Yoshihisa Yamamoto, and Ian R. Fisher Science 329, 824 (2010); M.A. Tartar, E. C. Blomberg, A. Kreyssig, M. G. Kim, N. Ni et al Phys. Rev. B 81, 184508 (2010); T.M. Chuang * T.-M. Chuang, M. P. Allan, J. Lee, Yang Xie et al, Science 327, 181 (2010); S. Kasahara et al, unpublished.

79 V. Stanev, J. Kang, Z. Tesanovic, Phys. Rev. B 78, 184509 (2008); V. Stanev, B. S. Alexandrov, P. Nikolić and Z. Tešanović, arXiv:1006.0447; V. Cvetkovic and Z. Tesanovic, Phys. Rev. B 80, 024512(2009). 
80 R. Thomale, C. Platt, W. Hanke, and B. A. Bernevig, Phys. Rev. Lett. 106, 187003 (2011).

81 T.A. Maier, S. Graser, D.J. Scalapino, and P.J. Hirschfeld, Phys. Rev. B 79224510 (2009).

82 R. Yu, P. Goswami, Q. Si, P. Nikolic, and J-X Zhu, arXiv:1103.3259.

83 A. Bernevig, private coimmunication.

84 T. Saito, S. Onari, and H. Kontani, Phys. Rev. B 83, 140512(R) (2011).

85 T.A. Maier, S. Graser, P. J. Hirschfeld, and D. J. Scalapino, Phys. Rev. B 83, 100515(R) (2011)

86 Fa. Wang, F. Yang, M. Gao, Z-Y. Lu, T. Xiang, and D-H. Lee. Europhys. Lett. 93, 57003 (2011).

87 R. Thomale, C. Platt, W. Hanke, J-P. Hu, and B. A. Bernevig, arXiv:1101.3593.

88 K. Suzuki, H. Usui, and K. Kuroki arXiv:1108.0657.

89 K. Nakayama, T. Sato, P. Richard, Y.-M. Xu, T. Kawahara, K. Umezawa, T. Qian, M. Neupane, G. F. Chen, H. Ding, and T. Takahashi, Phys. Rev. B 83, 020501 (2011); Y-M. Xu, Y-B. Huang, X-Y. Cui, E. Razzoli, M. Radovic et al Nature Physics 7, 198-202 (2011).

90 T. Kondo, A. F. Santander-Syro, O. Copie, C. Liu, M. E. Tillman, et al Phys. Rev. Lett. 101, 147003 (2008).

91 Y. Sekiba, T Sato, K Nakayama, K Terashima, P Richard et al New J. Phys. 11, 025020 (2009);

K. Terashima et. al., Proceedings of the National Academy of Sciences of the USA (PNAS) 106, $7330(2009)$.

92 C. Martin, M. E. Tillman, H. Kim, M. A. Tanatar, S. K. Kim et al Phys. Rev. Lett. 102, $247002(2009)$.

93 D.V. Efremov, M.M. Korshunov, O.V. Dolgov, A.A. Golubov, and P.J. Hirschfeld, arXiv:1104.3840.

94 A. D. Christianson, E. A. Goremychkin, R. Osborn, S. Rosenkranz, M. D. Lumsden, et. al., Nature 456, 930 (2008); R. Osborn, J.-P. Castellan, S. Rosenkranz, E. A. Goremychkin, D. Y. Chung, et al, arXiv:1106:0771.

95 see e.g. Ar. Abanov, A. V. Chubukov, and J. Schmalian Journal of Electron Spectroscopy and Related Phenomena, 117, 129 (2001); M. Eschrig, Adv. Phys. 55, 47 (2006), and references therein.

96 Y. Nagai and K. Kuroki, arXiv:1103.0586; T.A. Maier, S. Graser, P.J. Hirschfeld, D.J. Scalapino, Phys. Rev. B 83, 220505(R) (2011). 
97 S. Maiti, J. Knolle, I. Eremin, and A.V. Chubukov, arXiv:1108:0266

98 T. Hanaguri, S. Niitaka, K. Kuroki, and H. Takagi Science 328, 474 (2010).

99 I.I. Mazin amd D.J. Singh, arXiv 1007.0047; T. Hanaguri, S. Niitaka, K. Kuroki, and H. Takagi, arXiv:1007.0307.

100 R. M. Fernandes, D. K. Pratt, W. Tian, J. Zarestky, A. Kreyssig et. al., Phys. Rev. B 81, 140501(R) (2010); R.M. Fernandes and J. Schmalian, Phys. Rev. B 82, 014521 (2010); A.B.Vorontsov, M.G.Vavilov, and A.V.Chubukov, Phys. Rev. B 81, 174538 (2010); M. G. Vavilov, A. V. Chubukov, and A. B. Vorontsov, Supercond. Sci. Technol. 23, 054011 (2010).

101 J.-Ph. Reid, M. A. Tanatar, X. G. Luo, H. Shakeripour, S. René de Cotret et al . arXiv:1105.2232.

102 P. Popovich, A. V. Boris, O. V. Dolgov, A. A. Golubov, D. L. Sun, C. T. Lin, R. K. Kremer, and B. Keimer, Phys. Rev. Lett. 105, 027003 (2010).

103 C. Martin, R. T. Gordon, M. A. Tanatar, H. Kim, N. Ni, et al, Phys. Rev. B 80, 020501(R) (2009); R. Khasanov, D. V. Evtushinsky, A. Amato, H.-H. Klauss, H. Luetkens, et al Phys. Rev. Lett. 102, 187005(2009).

104 A.B. Vorontsov, M.G. Vavilov, and A.V. Chubukov, Phys. Rev. B 79, 140507(R) (2009); O.V. Dolgov, A.A. Golubov, D. Parker, New Journal of Physics, 11, 075012 (2009); Y. Bang, Europhys. Letters, 86, 47001 (2009).

105 H. Kim, R. T. Gordon, M. A. Tanatar, J. Hua, U. Welp et al Phys. Rev. B 82, 060518 (2010).

106 J.-Ph. Reid M. A. Tanatar, X. G. Luo, H. Shakeripour, N. Doiron-Leyraud et al Phys. Rev. B 82, 064501 (2010); M.A. Tanatar, J.-Ph. Reid, H. Shakeripour, X. G. Luo, N. Doiron-Leyraud et al, Phys. Rev. Lett. 104, 067002 (2010).

107 G.E. Volovik, JEPT Lett., 58, 469 (1993).

108 Y. Bang, Phys. Rev. Lett., 104, 217001 (2010).

109 K. Hashimoto, T. Shibauchi, T. Kato, K. Ikada, R. Okazaki et al, Phys. Rev. Lett. 102, 017002 (2009).

110 R.R. Gordon, H. Kim, N. Salovich, R. W. Giannetta, R. M. Fernandes et. al. L. Luan, T. M. Lippman, C. W. Hicks, J. A. Bert, O. M. Auslaender et. al., Phys. Rev. Lett. 106, 067001 (2011).

111 D-J. Jang, A. B. Vorontsov, I. Vekhter, K. Gofryk, Z. Yang et al, New Journal of Physics 13, 023036 (2011). 
112 B. Zeng, G. Mu, H. Q. Luo, T. Xiang, H. Yang et al, arXiv:1007.3597.

113 S.V. Borisenko, V. B. Zabolotnyy, D. V. Evtushinsky, T. K. Kim, I. V. Morozov et al Phys. Rev. Lett. 105, 067002 (2010).

114 D. S. Inosov, J. S. White, D. V. Evtushinsky, I. V. Morozov, A. Cameron et. al., Phys. Rev. Lett. 104, 187001 (2010).

115 H. Kim, M. A. Tanatar, Y. J. Song, Y. S. Kwon, and R. Prozorov, Phys. Rev. B 83, 100502 (2011).

116 L. Ma, J. Zhang, G. F. Chen, and W. Yu Phys. Rev. B 82, 180501(R) (2010)

117 D. Parker, M. G. Vavilov, A. V. Chubukov, and I. I. Mazin, Phys. Rev. B 80, 100508 (2009).

118 A.I. Coldea, J. D. Fletcher, A. Carrington, J. G. Analytis, A. F. Bangura, et al, Phys. Rev. Lett. 101, 216402 (2008).

119 S. Kasahara, T. Shibauchi, K. Hashimoto, K. Ikada, S. Tonegawa et al, Phys. Rev. B 81, 184519, (2010).

120 T. Shibauchi, private communication.

121 J.D. Fletcher, A. Serafin, L. Malone, J. G. Analytis, J.-H. Chu, et al Phys. Rev. Lett. 102, 147001 (2009); K. Hashimoto, A. Serafin, S. Tonegawa, R. Katsumata, R. Okazaki et al, Phys. Rev. B 82, 014526 (2010); M. Yamashita, N. Nakata, Y. Senshu, S. Tonegawa, K. Ikada, et al, Phys. Rev. B 80, 220509(R) (2009); J. S. Kim, P. J. Hirschfeld, G. R. Stewart, S. Kasahara, T. Shibauchi, T. Terashima, and Y. Matsuda, arXiv:1002.3355; Y. Nakai, T. Iye, S. Kitagawa, K. Ishida, H. Ikeda, et al, Phys. Rev. Lett. 105, 107003 (2010).

122 S. Maiti and A.V. Chubukov, arXiv:1104.2923.

123 M. Yamashita, Y. Senshu, T. Shibauchi, S. Kasahara, K. Hashimoto, et al, arXiv:1103.0885. 